\title{
WAVELET ESTIMATION OF THE LONG MEMORY PARAMETER FOR HERMITE POLYNOMIAL OF GAUSSIAN PROCESSES
}

\author{
M. CLAUSEL, F. ROUEFF, M. S. TAQQU, AND C. TUDOR
}

\begin{abstract}
We consider stationary processes with long memory which are non-Gaussian and represented as Hermite polynomials of a Gaussian process. We focus on the corresponding wavelet coefficients and study the asymptotic behavior of the sum of their squares since this sum is often used for estimating the long-memory parameter. We show that the limit is not Gaussian but can be expressed using the non-Gaussian Rosenblatt process defined as a Wiener-Itô integral of order 2. This happens even if the original process is defined through a Hermite polynomial of order higher than 2 .
\end{abstract}

\section{INTRODUCTION}

Wavelet analysis is a popular method for estimating the memory parameter of stochastic processes with long-range dependence. The idea of using wavelets to estimate the memory parameter $d$ goes back to [35] and [15, 16, 17, 18]. See also [2, 3], [5], [8], [7]. Wavelet methods are an alternative to the Fourier methods developed by Fox and Taqqu ([19]) and Robinson $([28,29])$. The case of the Gaussian processes, especially the fractional Brownian motion has been widely studied. In this paper we will make an analysis of the wavelet coefficients of stationary processes with long memory which are not Gaussian. The need for non-Gaussian self-similar processes in practice (for example in hydrology) is already mentioned in [33] based on the study of stochastic modeling for river-flow time series in [22]. More recently such an approach was used for modeling Internet traffic, see [32, Chapter 3 and 4].

The wavelet analysis of non-Gaussian stochastic processes has been much less treated in the literature. See [4] for some empirical studies. Bardet and Tudor, in [6], considered the case of the Rosenblatt process which is a non-Gaussian self-similar process with stationary increments living in the second Wiener chaos, that is, it can be expressed as a double iterated integral with respect to the Wiener process. It can be also defined as a Hermite process of order 2, while the fractional Brownian motion is a Hermite process of order 1 . We refer to Section 3 for the definition of the Rosenblatt process (see also [34], [1], [20]), and to [9], [10], [14], [34] for the definition and various properties of the Hermite process.

In the present work, we consider processes expressed as a Hermite polynomial of order greater than 1 of a Gaussian time series. This will allow us to gain insight into more complicated situations. A more general case, involving processes that can be expressed as (finite or

Date: November 19, 2018.

2010 Mathematics Subject Classification. Primary 42C40 ; 60G18 ; 62M15 ; Secondary: 60G20, 60 G22.

Key words and phrases. Hermite processes ; Wavelet coefficients ; Wiener chaos ; self-similar processes ; Long-range dependence.

F. Roueff's research was partially supported by the ANR project MATAIM NT09 441552.

Murad S.Taqqu was supported in part by the NSF grants DMS-0608669 and DMS-1007616 at Boston University.

C. Tudor's research was partially supported by the ANR grant Masterie BLAN 012103. 
infinite) sum of Hermite polynomials of a Gaussian time series is studied in our recent work 12]. In this work, we use :

a) a wide class of wavelets as in (2.6), instead of "variations";

b) an input process with long-range dependence, as in (2.2), instead of self-similar processes;

c) a semiparametric setup, as in (1.2), instead of a parametric one.

We derive the limit theorems that are needed for wavelet-based estimation procedures of the memory parameter. We will investigate the estimation problem in another paper.

Denote by $X=\left\{X_{t}\right\}_{t \in \mathbb{Z}}$ a centered stationary Gaussian process with unit variance and spectral density $f(\lambda), \lambda \in(-\pi, \pi)$. Such a stochastic process is said to have short memory or short-range dependence if $f(\lambda)$ is positive and bounded around $\lambda=0$ and long memory or long-range dependence if $f(\lambda) \rightarrow \infty$ as $\lambda \rightarrow 0$. We will suppose that $\left\{X_{t}\right\}_{t \in \mathbb{Z}}$ has long-memory with memory parameter $0<d<1 / 2$, that is,

$$
f(\lambda) \sim|\lambda|^{-2 d} f^{*}(\lambda) \text { as } \lambda \rightarrow 0
$$

where $f^{*}(\lambda)$ is a bounded spectral density which is continuous and positive at the origin. It is convenient to set

$$
f(\lambda)=\left|1-\mathrm{e}^{-\mathrm{i} \lambda}\right|^{-2 d} f^{*}(\lambda), \quad \lambda \in(-\pi, \pi] .
$$

Since the spectral density of a stationary process is integrable, we require $d<\frac{1}{2}$.

We shall also consider a process $\left\{Y_{t}\right\}_{t \in \mathbb{Z}}$, not necessarily stationary but its difference $\Delta^{K} Y$ of order $K \geq 0$ is stationary. Moreover, instead of supposing that $\Delta^{K} Y$ is Gaussian, we will assume that

$$
\left(\Delta^{K} Y\right)_{t}=H_{q_{0}}\left(X_{t}\right), \quad t \in \mathbb{Z},
$$

where $(\Delta Y)_{t}=Y_{t}-Y_{t-1}$, where $X$ is Gaussian with spectral density $f$ satisfying (1.2) and where $H_{q_{0}}$ is the $q_{0}$-th Hermite polynomial.

We will focus on the wavelet coefficients of $Y=\left\{Y_{t}\right\}_{t \in \mathbb{Z}}$. Since $\left\{Y_{t}\right\}_{t \in \mathbb{Z}}$ is random so will be its wavelet coefficients which we denote by $\left\{W_{j, k}, j \geq 0, k \in \mathbb{Z}\right\}$, where $j$ indicates the scale and $k$ the location. These wavelet coefficients are defined by

$$
W_{j, k}=\sum_{t \in \mathbb{Z}} h_{j}\left(\gamma_{j} k-t\right) Y_{t},
$$

where $\gamma_{j} \uparrow \infty$ as $j \uparrow \infty$ is a sequence of non-negative scale factors applied at scale $j$, for example $\gamma_{j}=2^{j}$ and $h_{j}$ is a filter whose properties will be listed below. We follow the engineering convention where large values of $j$ correspond to large scales. Our goal is to find the distribution of the empirical quadratic mean of these wavelet coefficients at large scales $j \rightarrow \infty$, that is, the asymptotic behavior of the scalogram

$$
S_{n, j}=\frac{1}{n} \sum_{k=0}^{n-1} W_{j, k}^{2},
$$

adequately normalized as the number of wavelet coefficients $n$ and $j=j(n) \rightarrow \infty$. This is a necessary and important step in developing methods for estimating the underlying long memory parameter $d$, see the references mentioned at the beginning of this section. Indeed, using the wavelet scalogram, there is standard way to construct an estimator of the memory parameter. The asymptotic behavior of the scalogram gives the convergence rate of this estimator. We provide more details in Section 5 .

When $q_{0}=1$, the behavior of $S_{n, j}$ has been studied in [31]. In this case, under certain conditions, the limit as $j, n \rightarrow \infty$ of the suitably renormalized sequence $S_{n, j}$ is Gaussian. If 
$q_{0} \geq 2$ only few facts are known on the behavior of the scalogram $S_{n, j}$. In [6], the authors have made a wavelet analysis of the Rosenblatt process (see Definition 3.1 with $q=2$ ). This situation roughly corresponds to the case $q_{0}=2$ (the second Hermite polynomial). It has been shown that its associated scalogram has a non-Gaussian behavior, that is, after normalization it converges to a Rosenblatt random variable. Basically, what happens is the following: the random variable $H_{2}\left(X_{t}\right)$ is, for every $t \in \mathbb{Z}$ an element of the second Wiener chaos and its square can be decomposed, using the properties of multiple stochastic integrals, as a sum of a multiple integral in the fourth Wiener chaos and a multiple integral in the second Wiener chaos. It turns out that the leading term is the one in the second Wiener chaos which converges to a Rosenblatt random variable (a Rosenblatt process at time 1). Wavelet analysis for $G=H_{q}$ with $q>2$ has not been done until now. Some intuition can be gained from the study of quadratic variations of the increments of the Hermite process, in [10]. In this case the starting process is self-similar, that is, invariant under scaling. Again the limit turns out to be the Rosenblatt random variable. Briefly since the Hermite process is an element of the $q$ th Wiener chaos, its square (minus the expectation of its square) can be expressed as a sum of multiple integrals of orders $2,4, \ldots$ until $2 q$. It turns out that the main term is the one in the second Wiener chaos which converges to a Rosenblatt random variable. This may suggest that in our situation one would have perhaps a "reduction theorem" as in [13], stating that it is the lower order term which dominates. This is not the case however. We will show in a subsequent paper that higher-order Hermite processes can appear in the limit even when the initial data are a mixture of a Gaussian and non-Gaussian components. See also [26], [25] for other examples of limit theorems based on the chaos expansion.

The paper is structured as follows. In Section 2 we introduce the wavelet filters and state the assumptions imposed on them. In Section 3 we state our main result and we introduce the Rosenblatt process which appears as limit for $q_{0} \geq 2$. This result is stated for a multivariate scalogram considered at a single scale. In Section 4, we explain how this applies to the asymptotic behavior of the univariate scalogram at multiple scales (in short, the multiscale asymptotics). Results on the estimation of the long memory parameter are derived in Section 5. In Section 6 we give the chaos expansion of the scalogram. Section 7 and 8 describe the asymptotic behavior of the main terms appearing in the decomposition of the scalogram. The proof of the main results is in Section 9, Finally, Sections A contains technical lemmas used throughout our paper and Appendix B recalls the basic facts needed in this paper about Wiener chaos.

\section{The WAVElet COEFficients}

The Gaussian sequence $X=\left\{X_{t}\right\}_{t \in \mathbb{Z}}$ with spectral density (1.2) is long-range dependent because $d>0$ and hence its spectrum explodes at $\lambda=0$. Whether $\left\{H_{q_{0}}\left(X_{t}\right)\right\}_{t \in \mathbb{Z}}$ is also long-range dependent depends on the respective values of $q_{0}$ and $d$. We show in [11], that the spectral density of $\left\{H_{q_{0}}\left(X_{t}\right)\right\}_{t \in \mathbb{Z}}$ behaves proportionally to $|\lambda|^{-\delta_{+}\left(q_{0}\right)}$ as $\lambda \rightarrow 0$, where

$$
\delta_{+}(q)=\max (\delta(q), 0) \quad \text { and } \quad \delta(q)=q d-(q-1) / 2, \quad q=1,2,3, \ldots,
$$

and hence $\delta_{+}\left(q_{0}\right)$ is the memory parameter of $\left\{H_{q_{0}}\left(X_{t}\right)\right\}_{t \in \mathbb{Z}}$. Therefore, since $0<d<1 / 2$, in order for $\left\{H_{q_{0}}\left(X_{t}\right)\right\}_{t \in \mathbb{Z}}, q_{0} \geq 1$, to be long-range dependent, one needs

$$
\delta\left(q_{0}\right)>0 \Leftrightarrow\left(1-1 / q_{0}\right) / 2<d<1 / 2
$$


that is, $d$ must be sufficiently close to $1 / 2$. Specifically, for long-range dependence,

$$
q_{0}=1 \Rightarrow d>0, \quad q_{0}=2 \Rightarrow d>1 / 4, \quad q_{0}=3 \Rightarrow d>1 / 3, \quad q_{0}=4 \Rightarrow d>3 / 8 \ldots
$$

From another perspective, for all $q_{0} \geq 1$

$$
\delta\left(q_{0}\right)>0 \Leftrightarrow q_{0}<1 /(1-2 d),
$$

and thus $\left\{H_{q_{0}}\left(X_{t}\right)\right\}_{t \in \mathbb{Z}}$ is short-range dependent if $q_{0} \geq 1 /(1-2 d)$. In the following, we always assume that $\left\{H_{q_{0}}\left(X_{t}\right)\right\}_{t \in \mathbb{Z}}$ has long memory, that is,

$$
1 \leq q_{0}<1 /(1-2 d) \text { or, equivalently, } 0<\delta\left(q_{0}\right)<1 / 2 .
$$

As indicated in the introduction, we consider the process $\left\{Y_{t}\right\}_{t \in \mathbb{Z}}$, where $\Delta^{K} Y_{t}=H_{q_{0}}\left(X_{t}\right)$ for any $t \in \mathbb{Z}$ and for some $K \geq 0$ (see (1.3) ). We are interested in the wavelets coefficients of the process $\left\{H_{q_{0}}\left(X_{t}\right)\right\}_{t \in \mathbb{Z}}$. To obtain them, one applies a linear filter $h_{j}(\tau), \tau \in \mathbb{Z}$, at each scale $j \geq 0$. We shall characterize below the filters $h_{j}(\tau)$ by their discrete Fourier transform :

$$
\widehat{h}_{j}(\lambda)=\sum_{\tau \in \mathbb{Z}} h_{j}(\tau) \mathrm{e}^{-\mathrm{i} \lambda \tau}, \lambda \in[-\pi, \pi], \quad h_{j}(\tau)=\frac{1}{2 \pi} \int_{-\pi}^{\pi} \widehat{h}_{j}(\lambda) \mathrm{e}^{\mathrm{i} \lambda \tau} \mathrm{d} \lambda, \tau \in \mathbb{Z} .
$$

The resulting wavelet coefficients $W_{j, k}$, where $j$ is the scale and $k$ the location are defined as

$$
W_{j, k}=\sum_{t \in \mathbb{Z}} h_{j}\left(\gamma_{j} k-t\right) Y_{t}=\sum_{t \in \mathbb{Z}} h_{j}\left(\gamma_{j} k-t\right) \Delta^{-K} H_{q_{0}}\left(X_{t}\right), j \geq 0, k \in \mathbb{Z},
$$

where $\gamma_{j} \uparrow \infty$ as $j \uparrow \infty$ is a sequence of non-negative scale factors applied at scale $j$, for example $\gamma_{j}=2^{j}$. We do not assume that the wavelet coefficients are orthogonal nor that they are generated by a multiresolution analysis, but only that the filters $h_{j}$ concentrate around the zero frequency as $j \rightarrow \infty$ with some uniformity, see Assumptions (W-b $)-(\mathrm{W}-\mathrm{c})$ below.

To study the joint convergence at several scales jointly going to infinity, wavelet coefficients can be considered as a process $W_{j+m_{0}, k}$ indexed by $m_{0}, k$ and where we let $j \rightarrow \infty$ as in [11]. Here we are interested in the scalogram defined as the empirical square mean (1.5) with $n$ equal to the number of wavelets coefficients at scale $j$ available from $N$ observations of the original process $Y_{1}, \ldots, Y_{N}$. Considering the joint asymptotic behavior at various scales means that we have to deal with different down-sampling rates $\gamma_{j}$ and different numbers $n_{j}$ of available wavelet coefficients, both indexed by the scale $j$. It is shown in [31] that the joint behavior of the scalogram at multiple scales can be deduced from the joint behavior of the statistic (1.5), viewed as a vector whose components have the same $j$ and $n$ but different filters $h_{\ell, j}, \ell=1, \ldots, m$. We shall adopt the multivariate scalogram setup in our asymptotic analysis. We shall apply it in Section 4 to deduce the multiscale asymptotic behavior of the univariate scalogram. This will also allow us to contrast the cases $q_{0}>1$ treated in this contribution with the case $q_{0}=1$ which follows from the result obtained in [30]. Our assumption on the filters $h_{\ell, j}, \ell=1, \ldots, m$ are the same as in [31, Theorem 1], except that we allow $\gamma_{j} \neq 2^{j}$ for the sake of generality, and we assume locally uniform convergence in the asymptotic behavior in (2.10). These assumptions are satisfied in the standard wavelet analysis described in [24] and briefly referred to in Section 4 .

From now on, the wavelet coefficient $W_{j, k}$ defined in (2.6) will be supposed to be $\mathbb{R}^{m}$-valued with $h_{j}$ representing a $m$-dimensional vector with entries $h_{\ell, j}, \ell=1, \ldots, m$. We will use bold 
faced symbols $\mathbf{W}_{j, k}$ and $\mathbf{h}_{j}$ to emphasize the multivariate setting, thus

$$
\mathbf{W}_{j, k}=\sum_{t \in \mathbb{Z}} \mathbf{h}_{j}\left(\gamma_{j} k-t\right) Y_{t}=\sum_{t \in \mathbb{Z}} \mathbf{h}_{j}\left(\gamma_{j} k-t\right) \Delta^{-K} H_{q_{0}}\left(X_{t}\right), j \geq 0, k \in \mathbb{Z} .
$$

We shall make the following assumptions on the filters $\mathbf{h}_{j}$ :

(W-a) Finite support: For each $\ell$ and $j,\left\{h_{\ell, j}(\tau)\right\}_{\tau \in \mathbb{Z}}$ has finite support.

(W-b) Uniform smoothness: There exists $M \geq 0, \alpha>1 / 2$ and $C>0$ such that for all $j \geq 0$ and $\lambda \in[-\pi, \pi]$

$$
\left|\widehat{\mathbf{h}}_{j}(\lambda)\right| \leq \frac{C \gamma_{j}^{1 / 2}\left|\gamma_{j} \lambda\right|^{M}}{\left(1+\gamma_{j}|\lambda|\right)^{\alpha+M}},
$$

where $|x|$ denotes the Euclidean norm of vector $x$. By $2 \pi$-periodicity of $\widehat{\mathbf{h}}_{j}$ this inequality can be extended to $\lambda \in \mathbb{R}$ as

$$
\left|\widehat{\mathbf{h}}_{j}(\lambda)\right| \leq C \frac{\gamma_{j}^{1 / 2}\left|\gamma_{j}\{\lambda\}\right|^{M}}{\left(1+\gamma_{j}|\{\lambda\}|\right)^{\alpha+M}},
$$

where $\{\lambda\}$ denotes the element of $(-\pi, \pi]$ such that $\lambda-\{\lambda\} \in 2 \pi \mathbb{Z}$.

(W-c) Asymptotic behavior: There exist a sequence of phase functions $\Phi_{j}: \mathbb{R} \rightarrow(-\pi, \pi]$ and some function $\widehat{\mathbf{h}}_{\infty}: \mathbb{R} \rightarrow \mathbb{C}^{p}$ such that

$$
\lim _{j \rightarrow+\infty} \gamma_{j}^{-1 / 2} \widehat{\mathbf{h}}_{j}\left(\gamma_{j}^{-1} \lambda\right) \mathrm{e}^{\mathrm{i} \Phi_{j}(\lambda)}=\widehat{\mathbf{h}}_{\infty}(\lambda),
$$

locally uniformly on $\lambda \in \mathbb{R}$.

In (W-C), locally uniformly means that for all $r>0$,

$$
\sup _{|\lambda| \leq r}\left|\gamma_{j}^{-1 / 2} \widehat{\mathbf{h}}_{j}\left(\gamma_{j}^{-1} \lambda\right) \mathrm{e}^{\mathrm{i} \Phi_{j}(\lambda)}-\widehat{\mathbf{h}}_{\infty}(\lambda)\right| \rightarrow 0
$$

This is satisfied if the set of filters correspond to a discrete wavelet transform (see Proposition 3 in [24]). Assumptions (2.8) and (2.10) imply that for any $\lambda \in \mathbb{R}$,

$$
\left|\widehat{\mathbf{h}}_{\infty}(\lambda)\right| \leq C \frac{|\lambda|^{M}}{(1+|\lambda|)^{\alpha+M}} .
$$

Hence vector $\widehat{\mathbf{h}}_{\infty}$ has entries in $L^{2}(\mathbb{R})$. We let $\mathbf{h}_{\infty}$ be the vector of $L^{2}(\mathbb{R})$ inverse Fourier transforms of $\widehat{h}_{\ell, \infty}, \ell=1, \ldots, m$, that is

$$
\widehat{\mathbf{h}}_{\infty}(\xi)=\int_{\mathbb{R}} \mathbf{h}_{\infty}(t) \mathrm{e}^{-i t \xi} \mathrm{d} t, \quad \xi \in \mathbb{R} .
$$

Observe that while $\widehat{\mathbf{h}}_{j}$ is $2 \pi$-periodic, the function $\widehat{\mathbf{h}}_{\infty}$ has non-periodic entries on $\mathbb{R}$. For the connection between these assumptions on $\mathbf{h}_{j}$ and corresponding assumptions on the scaling function $\varphi$ and the mother wavelet $\psi$ in the classical wavelet setting see [24] and [31]. In particular, in the univariate setting $m=1$, one has $\widehat{h}_{\infty}=\widehat{\varphi}(0) \overline{\widehat{\psi}}$.

For $M \geq K$, a more convenient way to express $\mathbf{W}_{j, k}$ is to incorporate the linear filter $\Delta^{-K}$ in (2.7) into the filter $\mathbf{h}_{j}$ and denote the resulting filter $\mathbf{h}_{j}^{(K)}$. Then

$$
\mathbf{W}_{j, k}=\sum_{t \in \mathbb{Z}} \mathbf{h}_{j}^{(K)}\left(\gamma_{j} k-t\right) H_{q_{0}}\left(X_{t}\right)
$$


where

$$
\widehat{\mathbf{h}}_{j}^{(K)}(\lambda)=\left(1-\mathrm{e}^{-\mathrm{i} \lambda}\right)^{-K} \widehat{\mathbf{h}}_{j}(\lambda)
$$

is the component wise discrete Fourier transform of $\mathbf{h}_{j}^{(K)}$. Since $\left\{H_{q_{0}}\left(X_{t}\right), t \in \mathbb{Z}\right\}$ is stationary, so is $\left\{W_{j, k}, k \in \mathbb{Z}\right\}$ for each scale $j$. Using (2.9), we further get,

$$
\left|\widehat{\mathbf{h}}_{j}^{(K)}(\lambda)\right| \leq C \gamma_{j}^{1 / 2+K} \frac{\left|\gamma_{j}\{\lambda\}\right|^{M-K}}{\left(1+\gamma_{j}|\{\lambda\}|\right)^{\alpha+M}}, \quad \lambda \in \mathbb{R}, j \geq 1 .
$$

In particular, if $M \geq K$, using that $\left(\left|\gamma_{j}\{\lambda\}\right| /\left(1+\gamma_{j}|\{\lambda\}|\right)\right)^{M} \leq\left(\left|\gamma_{j}\{\lambda\}\right| /\left(1+\gamma_{j}|\{\lambda\}|\right)\right)^{K}$, we get

$$
\left|\widehat{\mathbf{h}}_{j}^{(K)}(\lambda)\right| \leq C \gamma_{j}^{1 / 2+K}\left(1+\gamma_{j}|\{\lambda\}|\right)^{-\alpha-K}, \quad \lambda \in \mathbb{R}, j \geq 1 .
$$

By Assumption (2.8), $\mathbf{h}_{j}$ has vanishing moments up to order $M-1$, that is, for any integer $0 \leq k \leq M-1$,

$$
\sum_{t \in \mathbb{Z}} \mathbf{h}_{j}(t) t^{k}=0
$$

Observe that $\Delta^{K} Y$ is centered by definition. However, by (2.17), the definition of $\mathbf{W}_{j, k}$ only depends on $\Delta^{M} Y$. In particular, provided that $M \geq K+1$, its value is not modified if a constant is added to $\Delta^{K} Y$, whenever $M \geq K+1$.

\section{MAin RESUlt}

Recall that

$$
\left(\Delta^{K} Y\right)_{t}=H_{q_{0}}\left(X_{t}\right), \quad t \in \mathbb{Z} .
$$

The condition (2.4) ensures such that $\left\{H_{q_{0}}\left(X_{t}\right)\right\}_{t \in \mathbb{Z}}$ is long-range dependent (see [11], Lemma 4.1). Our main result deals with the asymptotic behavior of the scalogram $S_{n, j}$, defined in the univariate case $m=1$ by (1.5) as $j, n \rightarrow \infty$, that is, as $n \rightarrow \infty$ (large sample behavior) with $j=j(n)$ being an arbitrary diverging sequence (large scale behavior). More precisely, we will study the asymptotic behavior of the sequence

$$
\overline{\mathbf{S}}_{n, j}=\frac{1}{n} \sum_{k=0}^{n-1}\left(\mathbf{W}_{j, k}^{2}-\mathbb{E}\left[\mathbf{W}_{j, k}^{2}\right]\right)=\left[\frac{1}{n} \sum_{k=0}^{n-1}\left(W_{\ell, j, k}^{2}-\mathbb{E}\left[W_{\ell, j, k}^{2}\right]\right)\right]_{\ell=1, \ldots, m},
$$

adequately normalized as $j, n \rightarrow \infty$, where $W_{\ell, j, k}, \ell=1, \ldots, m$, denote the $m$ entries of vector $\mathbf{W}_{j, k}$. The limit will be expressed in terms of the Rosenblatt process which is defined as follows.

Definition 3.1. The Rosenblatt process of index $d$ with

$$
1 / 4<d<1 / 2,
$$

is the continuous time process

$$
Z_{d}(t)=\int_{\mathbb{R}^{2}}^{\prime \prime} \frac{\mathrm{e}^{\mathrm{i}\left(u_{1}+u_{2}\right) t}-1}{\mathrm{i}\left(u_{1}+u_{2}\right)}\left|u_{1}\right|^{-d}\left|u_{2}\right|^{-d} \mathrm{~d} \widehat{W}\left(u_{1}\right) \mathrm{d} \widehat{W}\left(u_{2}\right), t \in \mathbb{R} .
$$

The multiple integral (3.3) with respect to the complex-valued Gaussian random measure $\widehat{W}$ is defined in Appendix $\mathrm{B}$. The symbol $\int_{\mathbb{R}^{2}}^{\prime \prime}$ indicates that one does not integrate on the diagonal $u_{1}=u_{2}$. The integral is well-defined when (3.2) holds because then it has finite $L^{2}$ norm. This process is self-similar with self-similarity parameter

$$
H=2 d \in(1 / 2,1),
$$


that is for all $a>0,\left\{Z_{d}(a t)\right\}_{t \in \mathbb{R}}$ and $\left\{a^{H} Z_{d}(t)\right\}_{t \in \mathbb{R}}$ have the same finite dimensional distributions, see [34].

We now list the assumptions behind our main result:

Assumptions $\mathbf{A}\left\{\mathbf{W}_{j, k}, j \geq 1, k \in \mathbb{Z}\right\}$ are the wavelet coefficients defined by (2.7), where

(i) $X$ is a stationary Gaussian process with spectral density $f$ satisfying (1.2) with $0<d<$ $1 / 2$;

(ii) $H_{q_{0}}$ is the $q_{0}$ th Hermite polynomial where $q_{0}$ satisfies condition (2.4);

(iii) the sequence of positive integers $\left(\gamma_{j}\right)_{j>1}$ is non-decreasing and diverging;

(iv) the wavelet filters $\mathbf{h}_{j}=\left[h_{\ell, j}\right]_{\ell=1, \ldots, m}, j \geq 1$, satisfy (W-回)-(W-cl).

The definition of Hermite polynomials is recalled in Appendix B. The following theorem gives the limit of (3.1), suitably normalized, as the number of wavelet coefficients and the scale $j=j(n)$ tend to infinity, in the cases $q_{0}=1$ and $q_{0} \geq 2$.

Theorem 3.1. Suppose that Assumptions $\boldsymbol{A}$ hold with $M \geq K+\delta\left(q_{0}\right)$, where $\delta(\cdot)$ is defined in (2.1). Define the centered multivariate scalogram $\overline{\mathbf{S}}_{n, j}$ by (3.1) and let $\left(n_{j}\right)$ be any diverging sequence of integers.

(a) Suppose $q_{0}=1$ and that $\left(\gamma_{j}\right)$ is a sequence of even integers. Then, as $j \rightarrow \infty$,

$$
n_{j}^{1 / 2} \gamma_{j}^{-2(d+K)} \overline{\mathbf{S}}_{n_{j}, j} \stackrel{\mathcal{L}}{\longrightarrow} \mathcal{N}(0, \Gamma)
$$

where $\Gamma$ is the $m \times m$ matrix with entries

$$
\Gamma_{\ell, \ell^{\prime}}=4 \pi\left(f^{*}(0)\right)^{2} \int_{-\pi}^{\pi}\left|\sum_{p \in \mathbb{Z}}\right| \lambda+\left.\left.2 p \pi\right|^{-2(K+d)}\left[\widehat{h}_{\ell, \infty} \overline{\widehat{h}_{\ell^{\prime}, \infty}}\right](\lambda+2 p \pi)\right|^{2} \mathrm{~d} \lambda, \quad 1 \leq \ell, \ell^{\prime} \leq m .
$$

(b) Suppose $q_{0} \geq 2$. Then as $j \rightarrow \infty$,

$$
n_{j}^{1-2 d} \gamma_{j}^{-2\left(\delta\left(q_{0}\right)+K\right)} \overline{\mathbf{S}}_{n_{j}, j} \stackrel{\mathcal{L}}{\longrightarrow} f^{*}(0)^{q_{0}} \mathbf{L}_{q_{0}-1} Z_{d}(1)
$$

where $Z_{d}(1)$ is the Rosenblatt process in (3.3) evaluated at time $t=1, f^{*}(0)$ is the shortrange spectral density at zero frequency in (1.1) and where $\mathbf{L}_{q_{0}-1}$ is the deterministic $m$-dimensional vector $\left[L_{q_{0}-1}\left(\widehat{h}_{\ell, \infty}\right)\right]_{\ell=1, \ldots, m}$ with finite entries defined by

$$
L_{p}(g)=\int_{\mathbb{R}^{p}} \frac{\left|g\left(u_{1}+\cdots+u_{p}\right)\right|^{2}}{\left|u_{1}+\cdots+u_{p}\right|^{2 K}} \prod_{i=1}^{p}\left|u_{i}\right|^{-2 d} \mathrm{~d} u_{1} \cdots \mathrm{d} u_{p},
$$

for any $g: \mathbb{R} \rightarrow \mathbb{C}$ and $p \geq 1$.

This theorem is proved in Section 9,

Remark 3.1. Since $\delta(1)=d$ we observe that the exponent of $\gamma_{j}$ in the rate of convergence of $\overline{\mathbf{S}}_{n, j}$ can be written as $-2\left(\delta\left(q_{0}\right)+K\right)$ for both cases $q_{0}=1$ and $q_{0} \geq 2$, see (3.4) and (3.6), respectively. This corresponds to the fact that $d_{0}=\delta\left(q_{0}\right)+K$ is the long memory parameter of $Y$, and, as a consequence, $\mathbb{E}\left|\mathbf{W}_{j, 0}\right|^{2} \sim C \gamma_{j}^{2\left(\delta\left(q_{0}\right)+K\right)}$ as $j \rightarrow \infty$, see for example Theorem 5.1 in [11]. In contrast, the exponent of $n$ is always larger in the case $q_{0} \geq 2$, since this implies $2 d-1>-1 / 2$ under Condition (2.4). The statistical behavior of the limits are also very different in the two cases. In (3.4) the limit is Gaussian while in (3.6), the limit is Rosenblatt. 
M. CLAUSEL, F. ROUEFF, M. S. TAQQU, AND C. TUDOR

Another difference is that the entries of the limit vector in (3.6) have cross-correlations equal to 1 (they only differ through a multiplicative constant). In contrast, this typically does not happen in 3.4).

Remark 3.2. While $H_{q_{0}}\left(X_{t}\right)$ involves a single multiple integral of order $q_{0}, \mathbf{W}_{j, k}^{2}$ and hence $\overline{\mathbf{S}}_{n, j}$ in (3.1) involves a sum of multiple integrals of order 0, 2, 4, 6... up to $2 q_{0}$. But the limiting Rosenblatt process in Theorem 3.1 involves only a double integral, albeit with a nonrandom factor $\mathbf{L}_{q_{0}-1}$ expressed as a non-random multiple integral of order $q_{0}-1$. In view of Theorem 5.1 of [11], the components of $\mathbf{L}_{q_{0}-1}$ are the asymptotic variances of the wavelet coefficients applied to $\Delta^{-K} H_{q_{0}-1}\left(X_{t}\right)$.

\section{From multivariate to multiscale asymptotics}

Theorem 3.1 applies to multivariate filters $\mathbf{h}_{j}$ which define the scalogram $\mathbf{S}_{n, j}$. We will use it to obtain in Theorem 4.1 multiscale asymptotics for univariate filters and corresponding scalograms. This passage between these two prospectives is explained in the proof of Theorem 4.1. We use dyadic scales here, as in the standard wavelet analysis described in [24], where the wavelet coefficients are defined as

$$
W_{j, k}=\sum_{t \in \mathbb{Z}} g_{j}\left(2^{j} k-t\right) Y_{t},
$$

which corresponds to (1.4) with $\gamma_{j}=2^{j}$ and with $\left(g_{j}\right)$ denoting a sequence of filters that satisfies (W-国) $-(\mathrm{W}-\mathrm{c}$ ) with $m=1$, and $M$ and $\alpha$ respectively defined as the number of vanishing moments of the wavelet and its Fourier decay exponent. In the case of a multiresolution analysis, $g_{j}$ can be deduced from the associated mirror filters.

The number $n_{j}$ of wavelet coefficients available at scale $j$, is related both to the number $N$ of observations $Y_{1}, \cdots, Y_{N}$ of the time series $Y$ and to the length $T$ of the support of the wavelet $\psi$. More precisely, one has

$$
n_{j}=\left[2^{-j}(N-T+1)-T+1\right]=2^{-j} N+0(1),
$$

where $[x]$ denotes the integer part of $x$ for any real $x$. Details about the above facts can be found in $[24,31]$.

In this context, the scalogram is an empirical measure of the distribution of "energy of the signal" along scales, based on the $N$ observations $Y_{1}, \cdots, Y_{N}$. It is defined as

$$
\widehat{\sigma}_{j}^{2}=\frac{1}{n_{j}} \sum_{k=0}^{n_{j}-1} W_{j, k}^{2}, \quad j \geq 0,
$$

and is identical to $S_{n_{j}, j}$ defined in (1.5). Note that the sequence $\left(\widehat{\sigma}_{j}^{2}\right)_{j \geq 0}$ is indexed by the scale index $j$ but also depends on the number $N$ of observations through $n_{j}$. The wavelet spectrum is defined as

$$
\sigma_{j}^{2}=\mathbb{E}\left[\widehat{\sigma}_{j}^{2}\right]=\mathbb{E}\left[W_{j, k}^{2}\right] \text { for all } k,
$$

where the last equality holds for $M \geq K$ since in this case $\left\{W_{j, k}, k \in \mathbb{Z}\right\}$ is weakly stationary. We obtain the following result which provides asymptotics of the scalogram involving a finite number of different scales at the same time. We only provide the result for $q_{0} \geq 2$ since the case $q_{0}=1$ can be directly deduced from [31, Theorem 2]. 
Theorem 4.1. Suppose that Assumptions $\boldsymbol{A}$ (iㅣ)(iil) hold with $q_{0} \geq 2$. Set $\gamma_{j}=2^{j}$ and let $\left\{\left(g_{j}\right)_{j \geq 0}, g_{\infty}\right\}$ be a sequence of univariate filters satisfying $(W \square)-(W \mathrm{C})$ with $m=1$ and $M \geq \bar{\delta}\left(q_{0}\right)+K$. Then, as $j \rightarrow \infty$,

$$
\sigma_{j}^{2} \sim q_{0} !\left(f^{*}(0)\right)^{q_{0}} L_{q_{0}}\left(\widehat{g}_{\infty}\right) 2^{2 j\left(\delta\left(q_{0}\right)+K\right)} .
$$

Let now $j=j(N)$ be an increasing sequence such that $j \rightarrow \infty$ and $N 2^{-j} \rightarrow \infty$. Define $n_{j}$, $\widehat{\sigma}_{j}^{2}$ and $\sigma_{j}^{2}$ as in (4.2), (4.3) and (4.4), respectively. Then, as $N \rightarrow \infty$,

$$
\left\{n_{j}^{1-2 d}\left(\frac{\widehat{\sigma}_{j-u}^{2}}{\sigma_{j-u}^{2}}-1\right)\right\}_{u \geq 0} \stackrel{\text { fidi }}{\longrightarrow}\left\{2^{(2 d-1) u} \frac{L_{q_{0}-1}\left(\widehat{g}_{\infty}\right)}{q_{0} ! L_{q_{0}}\left(\widehat{g}_{\infty}\right)} Z_{d}(1)\right\}_{u \geq 0} .
$$

This theorem is proved in Section 9. Note that the constants $L_{q_{0}}\left(\widehat{g}_{\infty}\right)$ and $L_{q_{0}-1}\left(\widehat{g}_{\infty}\right)$ appearing in (4.5) and (4.6) are defined by (3.7). Here $\stackrel{\text { fidi }}{\longrightarrow}$ means the convergence of finitedimensional distributions, and since the limit depends on $u$ only through a deterministic multiplicative constant, we obtain, as in the multivariate case, that the multiscale limit has cross-correlations equal to 1.

As in the multivariate case, conveniently normalized, the centered multiscale scalogram is asymptotically a fully correlated Rosenblatt process. We recover the results of [6] where $Y$ is the Rosenblatt process itself. In other words Theorem 4 in [6] roughly corresponds here to the case $q_{0}=2$. The results in [10] correspond to the single scale limit for any $q_{0} \geq 2$, which indicate a limit of the scalogram (which corresponds to a wavelet large scale analysis) similar to that of the variogram (which corresponds to a small scale analysis using discrete variations).

\section{ESTIMATION OF THE LONG MEMORY PARAMETER}

We now consider the estimation of the long memory parameter of the observed process $\left\{Y_{t}\right\}_{t \in \mathbb{Z}}$ under the assumptions of Theorem 4.1, that are supposed to hold all along this section. As already mentioned, $\left\{H_{q_{0}}\left(X_{t}\right)\right\}_{t \in \mathbb{Z}}$ has long memory parameter $\delta\left(q_{0}\right)$. By (1.3), applying the setting of [24] for dealing with processes with stationary $K$-th increments, we get that $\left\{Y_{t}\right\}_{t \in \mathbb{Z}}$ itself has long memory parameter

$$
d_{0}=\delta\left(q_{0}\right)+K \text {. }
$$

We want to estimate this parameter from a sample $Y_{1}, \ldots, Y_{N}$. A typical wavelet estimator of $d_{0}$ reads

$$
\widehat{d_{0}}=\sum_{i=0}^{p-1} w_{i} \log \widehat{\sigma}_{j+i}^{2},
$$

where $w_{0}, \ldots, w_{p-1}$ are weights such that $w_{0}+\cdots+w_{p-1}=0$, and $\sum_{i=0}^{p-1} i w_{i}=1 /(2 \log 2)$, see [31]. Indeed, for this choice of weights and using (4.5) and (5.1), we see that, as $j \rightarrow \infty$,

$$
\begin{aligned}
\sum_{i=0}^{p-1} w_{i} \log \sigma_{j+i}^{2} & =\sum_{i=0}^{p-1} w_{i} \log \left(q_{0} !\left(f^{*}(0)\right)^{q_{0}} L_{q_{0}}\left(\widehat{g}_{\infty}\right) 2^{2 j d_{0}}\right)+d_{0}\left(\sum_{i=0}^{p-1} i w_{i}\right) 2 \log 2+o(1) \\
& =d_{0}+o(1) .
\end{aligned}
$$

Replacing $\sigma_{j+i}^{2}$ by $\widehat{\sigma}_{j+i}^{2}$ in the left-hand side of this approximation, we thus obtain an estimator $\widehat{d_{0}}$ of $d_{0}$. 
To obtain the asymptotic behavior of $\widehat{d_{0}}$ as $j$ and $N$ go to infinity, we first evaluate the bias, which is related to the approximation error in the equivalence (4.5). To this end, we must specify the convergence of $f^{*}(\lambda)$ to $f^{*}(0)$ as $\lambda \rightarrow 0$. A standard assumption in the semi-parametric setup is

$$
\left|f^{*}(\lambda)-f^{*}(0)\right| \leq C f^{*}(0)|\lambda|^{\beta} \quad \lambda \in(-\pi, \pi),
$$

where $\beta$ is some smoothness exponent in $(0,2]$. However here $f$ is the spectral density of the original Gaussian process $\left\{X_{t}\right\}$, hence we cannot apply directly the bound

$$
\left|\sigma_{j}^{2}-C_{1} 2^{2 d j}\right| \leq C_{2} 2^{(2 d-\beta) j},
$$

which corresponds to Relation (26) in Theorem 1 of [24] (with different notation for constants $C_{1}$ and $C_{2}$ ). In fact such a bound would contradict (4.5) since $d \neq d_{0}$, see (2.1) and (5.1). We must instead work with the (generalized) spectral density, say $\tilde{f}$, of the observed process $\left\{Y_{t}\right\}$. Applying Lemma 4.1 in [11], we have that the generalized spectral density $\tilde{f}$ of the process $\left\{Y_{t}\right\}=\left\{\Delta^{-K} H_{q_{0}}\left(X_{t}\right)\right\}$ satisfies

$$
\tilde{f}(\lambda)=q_{0} !\left|1-\mathrm{e}^{-\mathrm{i} \lambda}\right|^{-2 K} \underbrace{f \star \cdots \star f}_{q_{0} \text { times }}(\lambda),
$$

where $\star$ denotes periodic convolution. Now, by Lemma 8.2 in [11], we get

$$
q_{0} ! f \star \cdots \star f(\lambda)=\left|1-\mathrm{e}^{-\mathrm{i} \lambda}\right|^{-2 \delta\left(q_{0}\right)} \tilde{f}^{*}(\lambda),
$$

where $\tilde{f}^{*}$ denotes a nonnegative periodic function, continuous and positive at the origin, such that

$$
\left|\tilde{f}^{*}(\lambda)-\tilde{f}^{*}(0)\right| \leq C \tilde{f}^{*}(0)|\lambda|^{\tilde{\beta}} \quad \lambda \in(-\pi, \pi),
$$

where $\tilde{\beta}$ is any positive number such that $\tilde{\beta}<2 \delta\left(q_{0}\right)$ and $\tilde{\beta} \leq \beta$. Hence, we finally obtain

$$
\tilde{f}(\lambda)=\left|1-\mathrm{e}^{-\mathrm{i} \lambda}\right|^{-2 d_{0}} \tilde{f}^{*}(\lambda),
$$

and we may now apply Theorem 1 of [24] and use (4.5), to obtain that

$$
\left|\sigma_{j}^{2}-q_{0} !\left(f^{*}(0)\right)^{q_{0}} L_{q_{0}}\left(\widehat{g}_{\infty}\right) 2^{2 j d_{0}}\right| \leq C^{\prime} 2^{j\left(2 d_{0}-\tilde{\beta}\right)} .
$$

This yields

$$
\left|\sum_{i=0}^{p-1} w_{i} \log \sigma_{j+i}^{2}-d_{0}\right|=O\left(2^{-\tilde{\beta} j}\right),
$$

which is a more precise approximation than (5.3). Observe now that $\widehat{d_{0}}$ given in (5.2) satisfies the identity

$$
\widehat{d_{0}}=d_{0}+\sum_{i=0}^{p-1} w_{i} \log \left\{1+\left(\frac{\widehat{\sigma}_{j+i}^{2}}{\sigma_{j+i}^{2}}-1\right)\right\}+\sum_{i=0}^{p-1} w_{i} \log \sigma_{j+i}^{2}-d_{0} .
$$

Expanding $\log (x)$ in the neighborhood of $x=1$ and using (4.6) and (5.4), we obtain the following result.

Theorem 5.1. Suppose that the assumptions of Theorem 4.1 hold. As $N \rightarrow \infty$, if $j=j(N)$ is such that $j \rightarrow \infty$ and $N 2^{-j} \rightarrow \infty$, then

$$
\widehat{d_{0}}=d_{0}+n_{j}^{2 d-1} O_{P}(1)+O\left(2^{-\tilde{\beta} j}\right) .
$$


Moreover the $O_{P}$-term converges in distribution to the Rosenblatt variable

$$
\left(\sum_{i=0}^{p-1} w_{i} 2^{(1-2 d) i}\right) \frac{L_{q_{0}-1}\left(\widehat{g}_{\infty}\right)}{q_{0} ! L_{q_{0}}\left(\widehat{g}_{\infty}\right)} Z_{d}(1)
$$

To optimize the asymptotic term (5.5), one should choose weights $w_{0}, \ldots, w_{p-1}$ which minimize the constant in parentheses. It is interesting to note that this constant vanishes for some well-chosen weights, but that such a choice depends on the (unknown) parameter $d$. Observe also that the constant approaches 0 as $d$ approaches $1 / 2$, since $\sum_{i} w_{i}=0$.

Remark 5.1. To our knowledge, the non-linear semiparametric setting has not been considered before in this context. The closest reference appears to be [21], where the parametric Whittle estimator is studied for non-linear subordinated Gaussian processes. The comparison is difficult since, in the parametric approach of [21], the asymptotic results depend on the parameterization of the spectral density (essentially through the two constants $\rho_{1}$ and $\rho_{2}$ defined in [21]). However similarities can be observed in these results: the limit can be Rosenblatt, in which case the usual $n^{-1 / 2}$ parametric rate of convergence is replaced by $n^{2 d-1}$, see [21, Theorem 3.1] in the case $\rho_{1}=0$ and $\rho_{2} \neq 0$. This situation can be compared to Theorem 5.1 above, where the limit is also Rosenblatt and the usual $n_{j}^{-1 / 2}$ semiparametric rate is replaced by $n_{j}^{2 d-1}$. We thus expect that a semiparametric Whittle approach would have an asymptotic behavior similar to that of $\widehat{d_{0}}$.

\section{Chaos expansion of the scalogram}

Here we take $m=1$ without loss of generality, since the case $m \geq 2$ can be deduced by applying the case $m=1$ to each entry. The purpose of this section is to consider the scalogram $S_{n, j}$ defined in (1.5). and express it as a sum of multiple integrals $\widehat{I}(\cdot)$ (defined in Appendix A) with respect to the Gaussian random measure $\widehat{W}$. Our main tool will be the product formula for multiple Wiener-Itô integrals. In view of (B.7), $W_{j, k}$ is a multiple integral of order $q_{0}$ of some kernel $f_{j, k}$, that is

$$
W_{j, k}=\widehat{I}_{q_{0}}\left(f_{j, k}\right) \text {. }
$$

Now, using the product formula for multiple stochastic integrals (B.10), one gets, as shown in Proposition 6.1 that, for any $(n, j) \in \mathbb{N}^{2}$,

$$
S_{n, j}-\mathbb{E}\left(S_{n, j}\right)=\frac{1}{n} \sum_{k=0}^{n-1} W_{j, k}^{2}-\mathbb{E}\left[W_{j, 0}^{2}\right]=\sum_{p=0}^{q_{0}-1} p !\left(\begin{array}{c}
q_{0} \\
p
\end{array}\right)^{2} S_{n, j}^{(p)}
$$

where, for all $0 \leq p \leq q_{0}-1$,

$$
S_{n, j}^{(p)}=\widehat{I}_{2 q_{0}-2 p}\left(g_{p}\right) .
$$

That is, for every $j, n$, the random variable $S_{n, j}^{(p)}$ is an element of the chaos of order $2 q_{0}-2 p$. The function $g_{p}(\xi), \xi=\left(\xi_{1}, \ldots, \xi_{2 q_{0}-2 p}\right) \in \mathbb{R}^{2 q_{0}-2 p}$ is defined for every $p \in\left\{0, \cdots, q_{0}-1\right\}$ as

$$
g_{p}(\xi)=\frac{1}{n} \sum_{k=0}^{n-1}\left(f_{j, k} \bar{\otimes}_{p} f_{j, k}\right)
$$

where the contraction $\bar{\otimes}_{p}$ is defined in $(\underline{\mathrm{B} .11})$. 
Let us formalize the above decomposition of $S_{n, j}$ and give a more explicit expression for the function $g_{p}$ in (6.3).

Proposition 6.1. For all non-negative integer $j,\left\{W_{j, k}\right\}_{k \in \mathbb{Z}}$ is a weakly stationary sequence. Moreover, for any $(n, j) \in \mathbb{N}^{2}$,

$$
S_{n, j}-\mathbb{E}\left(S_{n, j}\right)=\sum_{p=0}^{q_{0}-1} p !\left(\begin{array}{c}
q \\
p
\end{array}\right)^{2} S_{n, j}^{(p)}
$$

where, for all $0 \leq p \leq q_{0}-1$,

$$
S_{n, j}^{(p)}=\widehat{I}_{2 q_{0}-2 p}\left(g_{p}\right)
$$

and where, for all $\xi=\left(\xi_{1}, \ldots, \xi_{2 q_{0}-2 p}\right) \in \mathbb{R}^{2 q_{0}-2 p}$,

$$
\begin{aligned}
g_{p}(\xi)= & D_{n}\left(\gamma_{j}\left(\xi_{1}+\cdots+\xi_{2 q_{0}-2 p}\right)\right) \\
& \times \prod_{i=1}^{2 q_{0}-2 p}\left[\sqrt{f\left(\xi_{i}\right)} \mathbb{1}_{(-\pi, \pi)}\left(\xi_{i}\right)\right] \times \widehat{\kappa}_{j}^{(p)}\left(\xi_{1}+\cdots+\xi_{q_{0}-p}, \xi_{q_{0}-p+1}+\cdots+\xi_{2 q_{0}-2 p}\right) .
\end{aligned}
$$

Here $f$ denotes the spectral density (1.2) of the underlying Gaussian process $X$ and

$$
D_{n}(u)=\frac{1}{n} \sum_{k=0}^{n-1} \mathrm{e}^{\mathrm{i} k u}=\frac{1-\mathrm{e}^{\mathrm{i} n u}}{n\left(1-\mathrm{e}^{\mathrm{i} u}\right)},
$$

denotes the normalized Dirichlet kernel. Finally, for $\xi=\left(\xi_{1}, \xi_{2}\right) \in \mathbb{R}^{2}$, if $p \neq 0$,

$$
\widehat{\kappa}_{j}^{(p)}\left(\xi_{1}, \xi_{2}\right)=\int_{(-\pi, \pi)^{p}}\left(\prod_{i=1}^{p} f\left(\lambda_{i}\right)\right) \widehat{h}_{j}^{(K)}\left(\lambda_{1}+\cdots+\lambda_{p}+\xi_{1}\right) \overline{\widehat{h}_{j}^{(K)}\left(\lambda_{1}+\cdots+\lambda_{p}-\xi_{2}\right)} \mathrm{d}^{p} \lambda
$$

and, if $p=0$,

$$
\widehat{\kappa}_{j}^{(p)}\left(\xi_{1}, \xi_{2}\right)=\widehat{h}_{j}^{(K)}\left(\xi_{1}\right) \overline{\widehat{h}_{j}^{(K)}\left(\xi_{2}\right)} .
$$

Notation. In (6.8), $\mathrm{d}^{p} \lambda$ refers to $p$-dimensional Lebesgue measure integration. To simplify the notation, we shall denote by $\Sigma_{q}$, the $\mathbb{C}^{q} \rightarrow \mathbb{C}$ function defined, for all $q \in \mathbb{Z}_{+}$and $y=\left(y_{1}, \ldots, y_{q}\right) \in \mathbb{C}^{q}$, by

$$
\Sigma_{q}(y)=\sum_{i=1}^{q} y_{i}
$$

and for any $\left(q_{1}, q_{2}\right) \in \mathbb{Z}_{+}^{2}$, we denote by $\Sigma_{q_{1}, q_{2}}$ the $\mathbb{C}^{q_{1}} \times \mathbb{C}^{q_{2}} \rightarrow \mathbb{C}^{2}$ function defined for all $y=\left(y_{1}, \ldots, y_{q_{1}+q_{2}}\right) \in \mathbb{C}^{q_{1}} \times \mathbb{C}^{q_{2}}$ by

$$
\Sigma_{q_{1}, q_{2}}(y)=\left(\sum_{i=1}^{q_{1}} y_{i}, \sum_{i=q_{1}+1}^{q_{2}} y_{i}\right) .
$$

With these notations, (6.5), (6.8) and (6.9) become respectively

$$
\begin{aligned}
& S_{n, j}^{(p)}=\widehat{I}_{2 q_{0}-2 p}\left(D_{n} \circ \Sigma_{2 q_{0}-2 p}\left(\gamma_{j} \times \cdot\right) \times\left[\sqrt{f} \mathbb{1}_{(-\pi, \pi)}\right]^{\otimes\left(2 q_{0}-2 p\right)} \times \widehat{\kappa}_{j}^{(p)} \circ \Sigma_{q_{0}-p, q_{0}-p}\right), \\
& \widehat{\kappa}_{j}^{(p)}\left(\xi_{1}, \xi_{2}\right)= \begin{cases}\int_{(-\pi, \pi)^{p}} f^{\otimes p}(\lambda) \widehat{h}_{j}^{(K)}\left(\Sigma_{p}(\lambda)+\xi_{1}\right) \overline{\widehat{h}_{j}^{(K)}\left(\Sigma_{p}(\lambda)-\xi_{2}\right)} \mathrm{d}^{p} \lambda & \text { if } p \neq 0, \\
{\left[\widehat{h}_{j}^{(K)} \otimes \widehat{h}_{j}^{(K)}\right]\left(\xi_{1}, \xi_{2}\right)} & \text { if } p=0,\end{cases}
\end{aligned}
$$


where $\circ$ denotes the composition of functions, $\lambda=\left(\lambda_{1}, \cdots, \lambda_{p}\right)$ and $f^{\otimes p}(\lambda)=f\left(\lambda_{1}\right) \cdots f\left(\lambda_{p}\right)$ is written as a tensor product.

Remark 6.1. The kernel $\widehat{\kappa}_{j}^{(p)}$ can also be expressed in terms of the the covariance sequence of the process $X$, namely,

$$
\widehat{\kappa}_{j}^{(p)}\left(\xi_{1}, \xi_{2}\right)=\sum_{m \in \mathbb{Z}^{2}} h_{j}^{(K)}\left(m_{1}\right) h_{j}^{(K)}\left(m_{2}\right) \mathbb{E}\left(X_{m_{2}} X_{m_{1}}\right)^{p} \mathrm{e}^{-\mathrm{i}\left(m_{1} \xi_{1}+m_{2} \xi_{2}\right)} .
$$

This follows from the relation

$$
\mathbb{E}\left(X_{m_{2}} X_{m_{1}}\right)=\int_{-\pi}^{\pi} e^{i\left(m_{2}-m_{1}\right) \lambda} f(\lambda) \mathrm{d} \lambda
$$

and 2.14) and the definition (2.5) of the discrete Fourier transform $\widehat{h}_{j}$.

Proof of Proposition 6.1 By (1.5),

$$
S_{n, j}=\frac{1}{n} \sum_{k=0}^{n-1} W_{j, k}^{2}
$$

Using ([6.1) and the product formula for multiple stochastic integrals (B.10) of Proposition B.1. we have

$$
W_{j, k}^{2}=\widehat{I}_{q_{0}}\left(f_{j, k}\right) \widehat{I}_{q_{0}}\left(f_{j, k}\right)=\sum_{p=0}^{q_{0}} p !\left(\begin{array}{c}
q_{0} \\
p
\end{array}\right)^{2} \widehat{I}_{2 q_{0}-2 p}\left(f_{j, k} \bar{\otimes}_{p} f_{j, k}\right)
$$

Therefore,

$$
S_{n, j}=\frac{1}{n} \sum_{k=0}^{n-1} W_{j, k}^{2}=\sum_{p=0}^{q_{0}} p !\left(\begin{array}{c}
q_{0} \\
p
\end{array}\right)^{2} \widehat{I}_{2 q_{0}-2 p}\left(g_{p}\right)
$$

where

$$
g_{p}=\frac{1}{n} \sum_{k=0}^{n-1} f_{j, k} \bar{\otimes}_{p} f_{j, k} .
$$

By (B.8), for all $\xi=\left(\xi_{1}, \cdots, \xi_{q_{0}}\right) \in \mathbb{R}^{q_{0}}$,

$$
f_{j, k}(\xi)=\exp \circ \Sigma_{q_{0}}\left(\mathrm{i} k \gamma_{j} \xi\right)\left(\widehat{h}_{j}^{(K)} \circ \Sigma_{q}(\xi)\right)\left(f^{\otimes q_{0}}(\xi)\right)^{1 / 2} \mathbb{1}_{(-\pi, \pi)}^{\otimes q_{0}}(\xi) .
$$

If, $p=1,2, \ldots, q_{0}-1$, let $\xi=\left(\xi_{1}, \cdots, \xi_{2 q_{0}-2 p}\right)$. The contraction $f_{j, k} \bar{\otimes}_{p} f_{j, k}$ defined on $\mathbb{R}^{2 q_{0}-2 p}$ equals by (B.11),

$$
\begin{aligned}
& f_{j, k} \bar{\otimes}_{p} f_{j, k}(\xi) \\
= & \int_{\mathbb{R}^{p}} f_{j, k}\left(\xi_{1}, \cdots, \xi_{q_{0}-p}, s\right) f_{j, k}\left(\xi_{q_{0}-p+1}, \cdots, \xi_{2 q_{0}-2 p},-s\right) \mathrm{d}^{p} s \\
= & \exp \circ \Sigma_{2 q_{0}-2 p}\left(\mathrm{i} k \gamma_{j} \xi\right) \times\left[\sqrt{f} \mathbb{1}_{(-\pi, \pi)}\right]^{\otimes 2 q_{0}-2 p}(\xi) \\
& \times \int_{\mathbb{R}^{p}} \widehat{h}_{j}^{(K)}\left(\xi_{1}+\cdots+\xi_{q_{0}-p}+\Sigma_{p}(\lambda)\right) \widehat{h}_{j}^{(K)}\left(\xi_{q_{0}-p+1}+\cdots+\xi_{2 q_{0}-2 p}-\Sigma_{p}(\lambda)\right) \times\left[f \mathbb{1}_{(-\pi, \pi)}\right]^{p}(\lambda) \mathrm{d}^{p} \lambda \\
= & \exp \circ \Sigma_{2 q_{0}-2 p}\left(\mathrm{i} k \gamma_{j} \xi\right) \times\left[\sqrt{f} \mathbb{1}_{(-\pi, \pi)}\right]^{\otimes 2 q_{0}-2 p}(\xi) \times \widehat{\kappa}_{j}^{(p)} \circ \Sigma_{q_{0}-p, q_{0}-p}(\xi),
\end{aligned}
$$


where $\widehat{\kappa}_{j}^{(p)}$ is defined by $(\underline{6.8}),(\underline{6.9})$, or equivalently by (6.13), (6.9) and where we used that $\overline{\widehat{h}_{j}^{(K)}(\cdot)}=\widehat{h}_{j}^{(K)}(-\cdot)$. We therefore get that $g_{p}$ is a function with $2 q_{0}-2 p$ variables given by

$$
g_{p}(\xi)=\frac{1}{n} \sum_{k=0}^{n-1} \exp \circ \Sigma_{2 q_{0}-2 p}\left(\mathrm{i} k \gamma_{j} \xi\right) \times\left[\sqrt{f} \mathbb{1}_{(-\pi, \pi)}\right]^{\otimes 2 q_{0}-2 p}(\xi) \times \widehat{\kappa}_{j}^{(p)} \circ \Sigma_{q_{0}-p, q_{0}-p}(\xi) .
$$

The Dirichlet kernel $D_{n}$ appears when one computes the sum $\frac{1}{n} \sum_{k=0}^{n-1} \exp \circ \Sigma_{2 q_{0}-2 p}\left(\mathrm{i} k \gamma_{j} \xi\right)$. This implies the formula (6.6).

The chaos of order zero does not appears in (6.4) where $S_{n, j}-\mathbb{E}\left(S_{n, j}\right)$ is considered. It appears however in the expression (6.17) of $S_{n, j}$ in the term with $p=q_{0}$ where $\widehat{I}_{2 q_{0}-2 p}=\widehat{I}_{0}$. In this case, we have

$$
q_{0} ! \widehat{I}_{0}\left(f_{j, k} \bar{\otimes}_{p} f_{j, k}\right)=q_{0} !\left\|f_{j, k}\right\|_{L^{2}\left(\mathbb{R}^{q_{0}}\right)}^{2}=\mathbb{E}\left(\left|W_{j, k}\right|^{2}\right),
$$

corresponding in (6.17) to the deterministic term

$$
\frac{1}{n} \sum_{k=1}^{n} \mathbb{E}\left(\left|W_{j, k}\right|^{2}\right)=\mathbb{E}\left(\left|W_{j, 0}\right|^{2}\right)=\mathbb{E}\left(S_{n, j}\right),
$$

by (6.15). Therefore $S_{n, j}-\mathbb{E}\left(S_{n, j}\right)$ can be expressed as (6.4).

As we can see from (6.4), the random variable $S_{n, j}$ can be expanded into a sum of multiple stochastic integrals starting from order zero (which corresponds to the deterministic term $\mathbb{E}\left(S_{n, j}\right)$ ). The order of the chaos appearing in the decomposition of $S_{n, j}$ could be greater or smaller than the critical value $1 /(1-2 d)$. This means that $S_{n, j}$ may admit summands with long-range dependence (orders smaller than $1 /(1-2 d)$ ) and short-range dependence (orders greater than $1 /(1-2 d)$ ). We will see that these two kind of terms have different behavior. Another issue concerns $p$, the order of the contraction in the product formula for multiple integrals. The case $p=0$ must be discussed separately because the function $\widehat{\kappa}_{j}^{(p)}$ in (6.8) has the special form (6.9) if $p=0$.

To study $S_{n, j}$ as $j, n \rightarrow \infty$, we need to study $S_{n, j}^{(p)}$ which is given in (6.12). We first estimate the $L^{2}$ norm of $S_{n, j}^{(p)}$.

\section{AN UPPER BOUND FOR THE $L^{2}$ NORM OF THE TERMS $S_{n, j}^{(p)}$}

To identify the leading term of the sum $S_{n, j}-\mathbb{E}\left(S_{n, j}\right)$, we will give an upper bound for the $L^{2}$ norms of the terms $S_{n, j}^{(p)} 0 \leq p<q_{0}$ defined in (6.5) and (6.12). Then, in Section 8 , we investigate the asymptotic behavior of the leading term of $S_{n, j}$. It directly implies the required result about the asymptotic bahavior of the scalogram. The expression (6.12) of $S_{n, j}^{(p)}$ involves the kernel $\widehat{\kappa}_{j}^{(p)}$ in (6.13) which vanishes when $\xi_{1}=0$ or $\xi_{2}=0$ if $p=0$ because $\widehat{h}_{j}(0)=0$ by (2.8). But the expression (6.13) of $\widehat{\kappa}_{j}^{(p)}$ implies that it does not vanish if $p>0$ because

$$
\widehat{\kappa}_{j}^{(p)}(0,0)=\int_{(-\pi, \pi)^{p}}\left(\prod_{i=1}^{p} f\left(\lambda_{i}\right)\right)\left|\widehat{h}_{j}\left(\Sigma_{p}(\lambda)\right)\right|^{2} \mathrm{~d}^{p} \lambda>0 .
$$

All these considerations lead one to distinguish the following two cases :

- The case $p \neq 0$.

- The case $p=0$. 
As for the Rosenblatt process considered by [6], the case $p=0$ requires different bounds and thus must be treated separately.

7.1. The case $p \neq 0$. Recall the expansion (6.4). In the case $p \neq 0$ we now give an upper bound of $\left\|S_{n, j}^{(p)}\right\|_{2}=\mathbb{E}\left(\left|S_{n, j}^{(p)}\right|^{2}\right)^{1 / 2}$ with $0<p<q_{0}<1 /(1-2 d)$.

Proposition 7.1. Let $0<p<q_{0}<1 /(1-2 d)$. There exists some $C>0$ whose value depends only on $p, d, q_{0}$ and $f^{*}$ such that for all $n, j \geq 2$

$$
\left\|S_{n, j}^{(p)}\right\|_{2} \leq C(\log n)^{\varepsilon} n^{-\min \left(1-2 \delta\left(q_{0}-p\right), 1 / 2\right)} \gamma_{j}^{2 \delta\left(q_{0}\right)+2 K},
$$

where $\varepsilon=1$ if $\delta\left(q_{0}-p\right)=1 / 4$ and $\varepsilon=0$ otherwise.

Proof. Let $C, C_{1}, \cdots$ be positive constants that may change from line to line. Set $r=q_{0}-p \geq$ 1. We perform the change of variable $y=n \gamma_{j} \xi$ in the integral expression of $S_{n, j}^{(p)}$ given by (6.12) and deduce that

$\mathbb{E}\left|S_{n, j}^{(p)}\right|^{2}=\frac{1}{\left(n \gamma_{j}\right)^{2 r}} \int_{\mathbb{R}^{2 r}}\left|D_{n} \circ \Sigma_{2 r}\left(\frac{y}{n}\right)\right|^{2}\left(\prod_{i=1}^{2 r}\left(f \mathbb{1}_{(-\pi, \pi)}\right)\left(\frac{y_{i}}{n \gamma_{j}}\right)\right)\left|\widehat{\kappa}_{j}^{(p)} \circ \Sigma_{r, r}\left(\frac{y}{n \gamma_{j}}\right)\right|^{2} \mathrm{~d}^{2 r} y$.

We now use the expression of $f$ given by (1.2), the boundedness of $f^{*}$, the bound of Dirichlet kernel given by Lemma A.3 and the bound of $\widehat{\kappa}_{j}^{(p)}$ given by Lemma A.1. Hence one deduces that there exists some $C_{1}>1$ depending only on $p, d$ such that

$$
\mathbb{E}\left|S_{n, j}^{(p)}\right|^{2} \leq C_{1} \gamma_{j}^{-2 r(1-2 d)} \gamma_{j}^{4(\delta(p)+K)} I_{n, j}=C_{1} \gamma_{j}^{-2+4 \delta(r)+4 \delta(p)} \gamma_{j}^{4 K} I_{n, j}
$$

where

$$
I_{n, j}=\int_{\left(-n \gamma_{j} \pi, n \gamma_{j} \pi\right)^{2 r}} \frac{n^{-2 r(1-2 d)}\left|g \circ \Sigma_{r, r}\left(\frac{y}{n \gamma_{j}}\right)\right|^{2} \mathrm{~d}^{2 r} y}{\left(1+n\left|\left\{\Sigma_{2 r}\left(n^{-1} y\right)\right\}\right|\right)^{2} \prod_{i=1}^{2 r}\left|y_{i}\right|^{2 d}}
$$

with

$$
g\left(z_{1}, z_{2}\right)=\frac{1}{\left(1+\gamma_{j}\left|\left\{z_{1}\right\}\right|\right)^{\delta(p)}\left(1+\gamma_{j}\left|\left\{z_{2}\right\}\right|\right)^{\delta(p)}} .
$$

We now bound the integral $I_{n, j}$. To this end, perform the successive change of variables

$$
u_{1}=\frac{y_{1}+\cdots+y_{r}}{n}, \cdots, u_{r}=\frac{y_{r}}{n}, v_{1}=\frac{y_{r+1}+\cdots+y_{2 r}}{n}, \cdots, v_{r}=\frac{y_{2 r}}{n},
$$

so that

$$
\begin{array}{r}
y_{i}=n\left(u_{i}-u_{i+1}\right) \text { for } 1 \leq i \leq r-1, y_{r}=n u_{r}, \\
y_{i}=n\left(v_{i-r}-v_{i-r+1}\right) \text { for } r+1 \leq i \leq 2 r-1, y_{2 r}=n u_{r} .
\end{array}
$$

In addition, observe that for any $m \in \mathbb{Z}_{+} \backslash\{0\},\left(y_{1}, \cdots, y_{m}\right) \in\left(-n \gamma_{j} \pi, n \gamma_{j} \pi\right)^{m}$, implies that $y_{1}+\cdots+y_{m} \in\left(-m\left(n \gamma_{j}\right) \pi, m\left(n \gamma_{j}\right) \pi\right)$. Hence, there exists some constant $C$ depending only on $r, d$ such that

$$
I_{n, j} \leq C \int_{-\gamma_{j} \pi r}^{\gamma_{j} \pi r} \int_{-\gamma_{j} \pi r}^{\gamma_{j} \pi r} \frac{J_{r, \gamma_{j} \pi}\left(u_{1} ; 2 d 1_{r}\right) J_{r, \gamma_{j} \pi}\left(v_{1} ; 2 d 1_{r}\right) \mathrm{d} u_{1} \mathrm{~d} v_{1}}{\left(1+n\left|\left\{u_{1}+v_{1}\right\}\right|\right)^{2}\left(1+\gamma_{j}\left|\left\{\frac{u_{1}}{\gamma_{j}}\right\}\right|\right)^{2 \delta(p)}\left(1+\gamma_{j}\left|\left\{\frac{v_{1}}{\gamma_{j}}\right\}\right|\right)^{2 \delta(p)}},
$$

where we used the definition of $J_{m, a}(s ; \beta)$ in Lemma A.6 with the notation $1_{r}$ for the $r$ dimensional vector with all entries equal to 1 , that is, we set $m=r, a=\gamma_{j} \pi, \beta_{1}=\cdots=$ 
$\beta_{m}=2 d$ in (A.14). We now apply Lemma A.6. Since $m=r<1 /(1-2 d)$, we are in Case (i) and we get that there exists some $C>0$ depending only on $r, d$ such that

$$
J_{r, \gamma_{j} \pi}\left(s ; 2 d 1_{r}\right) \leq C|s|^{-2 \delta(r)} \text { for all } s \in \mathbb{R} .
$$

Then there exists some constant $C_{2}>1$ depending only on $r, d$ such that

$$
I_{n, j} \leq C_{2} \int_{-\gamma_{j} \pi r}^{\gamma_{j} \pi r} \int_{-\gamma_{j} \pi r}^{\gamma_{j} \pi r} \frac{\left|u_{1}\right|^{-2 \delta(r)}\left|v_{1}\right|^{-2 \delta(r)} \mathrm{d} u_{1} \mathrm{~d} v_{1}}{\left(1+n\left|\left\{u_{1}+v_{1}\right\}\right|\right)^{2}\left(1+\gamma_{j}\left|\left\{\frac{u_{1}}{\gamma_{j}}\right\}\right|\right)^{2 \delta(p)}\left(1+\gamma_{j}\left|\left\{\frac{v_{1}}{\gamma_{j}}\right\}\right|\right)^{2 \delta(p)}} .
$$

Now use the inequality $|\{x\}| \leq|x|$ valid on $x \in \mathbb{R}$. Since $\delta(r) \geq 0$,

$$
I_{n, j} \leq C_{2} \int_{-\gamma_{j} \pi r}^{\gamma_{j} \pi r} \int_{-\gamma_{j} \pi r}^{\gamma_{j} \pi r} \frac{\left|\gamma_{j}\left\{\frac{u_{1}}{\gamma_{j}}\right\}\right|^{-2 \delta(r)}\left|\gamma_{j}\left\{\frac{v_{1}}{\gamma_{j}}\right\}\right|^{-2 \delta(r)} \mathrm{d} u_{1} \mathrm{~d} v_{1}}{\left(1+n\left|\left\{u_{1}+v_{1}\right\}\right|\right)^{2}\left(1+\gamma_{j}\left|\left\{\frac{u_{1}}{\gamma_{j}}\right\}\right|\right)^{2 \delta(p)}\left(1+\gamma_{j}\left|\left\{\frac{v_{1}}{\gamma_{j}}\right\}\right|\right)^{2 \delta(p)}} .
$$

By $2 \pi$-periodicity of $x \mapsto\{x\}$, the integrand is $\left(2 \gamma_{j} \pi\right)$-periodic with respect to both variables $u_{1}$ and $v_{1}$ and we get that

$$
I_{n, j} \leq C_{3} \int_{-\gamma_{j} \pi}^{\gamma_{j} \pi} \int_{-\gamma_{j} \pi}^{\gamma_{j} \pi} \frac{\left|u_{1}\right|^{-2 \delta(r)}\left|v_{1}\right|^{-2 \delta(r)} \mathrm{d} u_{1} \mathrm{~d} v_{1}}{\left(1+n\left|\left\{u_{1}+v_{1}\right\}\right|\right)^{2}\left(1+\left|u_{1}\right|\right)^{2 \delta(p)}\left(1+\left|v_{1}\right|\right)^{2 \delta(p)}} .
$$

To deal with the fractional parts, we now partition $\left(-\gamma_{j} \pi, \gamma_{j} \pi\right)^{2}$ using the following domains

$$
\Delta_{j}^{(s)}=\left\{\left(u_{1}, v_{1}\right) \in\left(-\gamma_{j} \pi, \gamma_{j} \pi\right)^{2},\left|u_{1}+v_{1}-2 \pi s\right| \leq \pi\right\}
$$

with $s \in\left\{-\gamma_{j}, \ldots, \gamma_{j}\right\}$, so that $I_{n, j}=A+2 B$ with

$$
A=\int_{\Delta_{j}^{(0)}} \frac{\left|u_{1}\right|^{-2 \delta(r)}\left|v_{1}\right|^{-2 \delta(r)} \mathrm{d} u_{1} \mathrm{~d} v_{1}}{\left(1+n\left|u_{1}+v_{1}\right|\right)^{2}\left(1+\left|u_{1}\right|\right)^{2 \delta(p)}\left(1+\left|v_{1}\right|\right)^{2 \delta(p)}},
$$

and

$$
B=\sum_{s=1}^{\gamma_{j}} \int_{\Delta_{j}^{(s)}} \frac{\left|u_{1}\right|^{-2 \delta(r)}\left|v_{1}\right|^{-2 \delta(r)} \mathrm{d} u_{1} \mathrm{~d} v_{1}}{\left(1+n\left|u_{1}+v_{1}-2 \pi s\right|\right)^{2}\left(1+\left|u_{1}\right|\right)^{2 \delta(p)}\left(1+\left|v_{1}\right|\right)^{2 \delta(p)}} .
$$

Let us now bound separately $A$ and $B$. To bound $A$, we distinguish two cases : $4 \delta(r)>1$ and $4 \delta(r) \leq 1$. In the first case, observe that $(1+|u|)^{2 \delta(p)} \geq 1$ holds on $\mathbb{R}$ and perform the change of variables $u_{1}^{\prime}=n u_{1}$ and $v_{1}^{\prime}=n v_{1}$. Then

$$
A \leq n^{-2+4 \delta(r)} \int_{\mathbb{R}^{2}} \frac{\left|u_{1}^{\prime}\right|^{-2 \delta(r)}\left|v_{1}^{\prime}\right|^{-2 \delta(r)} \mathrm{d} u_{1}^{\prime} \mathrm{d} v_{1}^{\prime}}{\left(1+\left|u_{1}^{\prime}+v_{1}^{\prime}\right|\right)^{2}} \leq C n^{-2+4 \delta(r)},
$$

since the integral is bounded. This follows from Lemma 8.4 of [11] applied with $M_{1}=2$, $M_{2}=0, q=2, a=0, \beta_{1}=\beta_{2}=2 \delta(r)$.

In the case where $4 \delta(r) \leq 1$, setting $t_{1}=u_{1}+v_{1}$, we get that

$$
A \leq \int_{-\pi}^{-\pi} \frac{\mathrm{d} t_{1}}{\left(1+n\left|t_{1}\right|\right)^{2}}\left[\int_{-\gamma_{j} \pi}^{\gamma_{j} \pi} \frac{\left|t_{1}-v_{1}\right|^{-2 \delta(r)}\left|v_{1}\right|^{-2 \delta(r)} \mathrm{d} v_{1}}{\left(1+\left|t_{1}-v_{1}\right|\right)^{2 \delta(p)}\left(1+\left|v_{1}\right|\right)^{2 \delta(p)}}\right] .
$$

We now split the integral in brackets into two terms

$$
\int_{\left|v_{1}\right| \leq 2\left|t_{1}\right|} \frac{\left|t_{1}-v_{1}\right|^{-2 \delta(r)}\left|v_{1}\right|^{-2 \delta(r)} \mathrm{d} v_{1}}{\left(1+\left|t_{1}-v_{1}\right|\right)^{2 \delta(p)}\left(1+\left|v_{1}\right|\right)^{2 \delta(p)}}+\int_{2\left|t_{1}\right| \leq\left|v_{1}\right| \leq \gamma_{j} \pi} \frac{\left|t_{1}-v_{1}\right|^{-2 \delta(r)}\left|v_{1}\right|^{-2 \delta(r)} \mathrm{d} v_{1}}{\left(1+\left|t_{1}-v_{1}\right|\right)^{2 \delta(p)}\left(1+\left|v_{1}\right|\right)^{2 \delta(p)}}
$$


Consider the first integral. Since $4 \delta(r) \leq 1$, Lemma A.6 (case (ii) or (iv)) applied with $m=2$, $a=2\left|t_{1}\right|, s_{1}=t_{1}, \beta_{1}=\beta_{2}=2 \delta(r)$ then implies that for some $C>0$ depending on $r, d$

$$
\begin{aligned}
\int_{\left|v_{1}\right| \leq 2\left|t_{1}\right|} \frac{\left|t_{1}-v_{1}\right|^{-2 \delta(r)}\left|v_{1}\right|^{-2 \delta(r)} \mathrm{d} v_{1}}{\left(1+\left|t_{1}-v_{1}\right|\right)^{2 \delta(p)}\left(1+\left|v_{1}\right|\right)^{2 \delta(p)}} & \leq \int_{\left|v_{1}\right| \leq 2\left|t_{1}\right|}\left|t_{1}-v_{1}\right|^{-2 \delta(r)}\left|v_{1}\right|^{-2 \delta(r)} \mathrm{d} v_{1} \\
& \leq C\left|t_{1}\right|^{1-4 \delta(r)} .
\end{aligned}
$$

Now consider the second integral. Note that $\left|v_{1}\right| \geq 2\left|t_{1}\right|$ implies $\left|v_{1}-t_{1}\right| \geq\left|v_{1}\right|-\left|t_{1}\right| \geq\left|v_{1}\right| / 2$. We get that

$$
\begin{aligned}
\int_{2\left|t_{1}\right| \leq\left|v_{1}\right| \leq \gamma_{j} \pi} \frac{\left|t_{1}-v_{1}\right|^{-2 \delta(r)}\left|v_{1}\right|^{-2 \delta(r)} \mathrm{d} v_{1}}{\left(1+\left|t_{1}-v_{1}\right|\right)^{2 \delta(p)}\left(1+\left|v_{1}\right|\right)^{2 \delta(p)}} & \leq C \int_{2\left|t_{1}\right|}^{\gamma_{j} \pi} \frac{\left|v_{1}\right|^{-2 \delta(r)}\left|v_{1}\right|^{-2 \delta(r)} \mathrm{d} v_{1}}{\left(1+\left|v_{1}\right|\right)^{2 \delta(p)}\left(1+\left|v_{1}\right|\right)^{2 \delta(p)}} \\
& \leq C \int_{2\left|t_{1}\right|}^{\gamma_{j} \pi} \frac{\left|v_{1}\right|^{-4 \delta(r)} \mathrm{d} v_{1}}{\left(1+\left|v_{1}\right|\right)^{4 \delta(p)}} \\
& =O\left(\left(1+|\log | t_{1}||\right)^{\varepsilon}\right),
\end{aligned}
$$

where we used that $-4 \delta(r) \geq-1$ with equality if and only if $\varepsilon=1$ and that $4(\delta(r)+\delta(p))=$ $4 \delta\left(q_{0}\right)+2>2$. Hence, if $4 \delta(r) \leq 1$

$$
A \leq C\left(\int_{-\pi}^{\pi} \frac{\left(1+|\log | t_{1}||\right)^{\varepsilon} \mathrm{d} t_{1}}{\left(1+n\left|t_{1}\right|\right)^{2}}\right) \leq C n^{-1}(\log n)^{\varepsilon} .
$$

To sum up Equations (7.6) and (7.7), we can write

$$
A \leq C(\log n)^{\varepsilon} n^{-\min (2-4 \delta(r), 1)} .
$$

To bound $B$ observe that, on $\mathbb{R}^{2}$, if $\left|u_{1}\right| \leq\left|u_{1}+v_{1}\right| / 2$ then

$$
\left|v_{1}\right|=\left|\left(u_{1}+v_{1}\right)-u_{1}\right| \geq\left|u_{1}+v_{1}\right|-\left|u_{1}\right| \geq\left|u_{1}+v_{1}\right| / 2 .
$$

Hence either $\left|u_{1}\right| \geq\left|u_{1}+v_{1}\right| / 2$ or $\left|v_{1}\right| \geq\left|u_{1}+v_{1}\right| / 2$. Set

$$
\Delta_{j}^{(s, 1)}=\left\{\left(u_{1}, v_{1}\right) \in \Delta_{j}^{(s)},\left|u_{1}\right| \geq\left|u_{1}+v_{1}\right| / 2\right\},
$$

and its symmetric set

$$
\Delta_{j}^{(s, 2)}=\left\{\left(u_{1}, v_{1}\right) \in \Delta_{j}^{(s)},\left|v_{1}\right| \geq\left|u_{1}+v_{1}\right| / 2\right\} .
$$

Then, since $\delta(r), \delta(p)>0$, for any $s \in\left\{-\gamma_{j}, \cdots,-1,1, \cdots, \gamma_{j}\right\}$,

$$
\begin{aligned}
B^{(s, 1)} & =\int_{\Delta_{j}^{(s, 1)}} \frac{\left|u_{1}\right|^{-2 \delta(r)}\left|v_{1}\right|^{-2 \delta(r)} \mathrm{d} u_{1} \mathrm{~d} v_{1}}{\left(1+n\left|\left\{u_{1}+v_{1}\right\}\right|\right)^{2}\left(1+\left|u_{1}\right|\right)^{2 \delta(p)}\left(1+\left|v_{1}\right|\right)^{2 \delta(p)}} \\
& \leq C \int_{\Delta_{j}^{(s, 1)}} \frac{\left|u_{1}\right|^{-2(\delta(r)+\delta(p))}\left|v_{1}\right|^{-2 \delta(r)} \mathrm{d} u_{1} \mathrm{~d} v_{1}}{\left(1+n\left|\left\{u_{1}+v_{1}\right\}\right|\right)^{2}\left(1+\left|v_{1}\right|\right)^{2 \delta(p)}} \\
& \leq C \int_{\Delta_{j}^{(s, 1)}} \frac{\left|u_{1}+v_{1}\right|^{-2(\delta(r)+\delta(p))}\left|v_{1}\right|^{-2 \delta(r)} \mathrm{d} u_{1} \mathrm{~d} v_{1}}{\left(1+n\left|\left\{u_{1}+v_{1}\right\}\right|\right)^{2}\left(1+\left|v_{1}\right|\right)^{2 \delta(p)}} .
\end{aligned}
$$

Setting $t_{1}=n\left(u_{1}+v_{1}\right)$, we get that

$$
B^{(s, 1)} \leq C n^{-1+2 \delta(r)+2 \delta(p)}\left(\int_{t_{1}=2 \pi n s-\pi n}^{2 \pi n s+\pi n} \frac{\left|t_{1}\right|^{-2 \delta(r)-2 \delta(p)} \mathrm{d} t_{1}}{\left(1+\left|t_{1}-2 \pi n s\right|\right)^{2}}\right)\left(\int_{-\gamma_{j} \pi}^{\gamma_{j} \pi} \frac{\left|v_{1}\right|^{-2 \delta(r)} \mathrm{d} v_{1}}{\left(1+\left|v_{1}\right|\right)^{2 \delta(p)}}\right) .
$$


Set $w_{1}=t_{1}-2 \pi n s$. Since $s \neq 0$, we have

$$
B^{(s, 1)} \leq C n^{-1+2 \delta(r)+2 \delta(p)}(n(2|s|-1))^{-2 \delta(r)-2 \delta(p)}\left(\int_{\mathbb{R}}\left(1+\left|w_{1}\right|\right)^{-2} \mathrm{~d} w_{1}\right)\left(\int_{-\gamma_{j} \pi}^{\gamma_{j} \pi} \frac{\left|v_{1}\right|^{-2 \delta(r)} \mathrm{d} v_{1}}{\left(1+\left|v_{1}\right|\right)^{2 \delta(p)}}\right),
$$

and the same bound holds on $B^{(s, 2)}$ by symmetry. Hence

$$
B=\sum_{s=1}^{\gamma_{j}}\left(B^{(s, 1)}+B^{(s, 2)}\right) \leq C n^{-1}\left(\sum_{|s|=1}^{\gamma_{j}}(2|s|-1)^{-2 \delta(r)-2 \delta(p)}\right)\left(\int_{-\gamma_{j} \pi}^{\gamma_{j} \pi} \frac{\left|v_{1}\right|^{-2 \delta(r)} \mathrm{d} v_{1}}{\left(1+\left|v_{1}\right|\right)^{2 \delta(p)}}\right) .
$$

Using $2 \delta(p)+2 \delta(r)=\delta\left(q_{0}\right)+1>1$, we deduce from (17.9) that $B=O\left(n^{-1}\right)$ and, with (7.8), $I_{n, j}=A+B=O\left((\log n)^{\varepsilon} n^{-\min (2-4 \delta(r), 1)}\right)$. With $(\overline{7.2})$ and $\delta(p)+\delta(r)=\delta\left(q_{0}\right)+1 / 2$, we obtain (7.1).

7.2. The case $p=0$. Here the situation is different from the previous case $p \neq 0$ since the kernel $\widehat{\kappa}_{j}^{(p)}$ involved in the definition of $S_{n, j}^{(p)}$ has a different expression when $p=0$ and vanishes when $\xi_{1}=0$ or $\xi_{2}=0$. It implies that the bound in Proposition 7.2 involves $n^{-1 / 2}$ instead of $n^{-1+\delta\left(q_{0}\right)}$ as could be expected from the case $p>0$ in Proposition 7.1. Further, an additional assumption on the moments of the wavelet is required which is consistent with the results proved in the Gaussian case in [24] (corresponding to $q_{0}=1$ ) where $M$ is assumed to be greater than $K+d$.

Proposition 7.2. Assume that $M \geq \delta\left(q_{0}\right)+K$. Then there exists some $C>1$ whose values depend only on $q_{0}, d$ such that for any $n, j$

$$
\left\|S_{n, j}^{(0)}\right\|_{L^{2}(\Omega)}=\mathbb{E}\left(\left|S_{n, j}^{(0)}\right|^{2}\right)^{1 / 2} \leq C n^{-1 / 2} \gamma_{j}^{2 \delta\left(q_{0}\right)+2 K} .
$$

Proof. We denote by $C$ a positive constant that may change at each appearance, but whose value does neither depend on $n$ nor $j$. Since $p=0, \widehat{\kappa}_{j}^{(0)}=\widehat{h}_{j}^{(K) \otimes 2}$ by (6.9). Then, setting $y=\left(n \gamma_{j}\right)^{-1} \xi$ in (6.12), we get

$$
\begin{aligned}
& \mathbb{E}\left|S_{n, j}^{(0)}\right|^{2} \\
& =\frac{1}{\left(n \gamma_{j}\right)^{2 q_{0}}} \int_{\mathbb{R}^{2 q_{0}}}\left|D_{n} \circ \Sigma_{2 q_{0}}\left(\frac{y}{n}\right)\right|^{2}\left(f \mathbb{1}_{(-\pi, \pi)}\right) \otimes\left(2 q_{0}\right)\left(\frac{y}{n \gamma_{j}}\right)\left|\widehat{h}_{j}^{(K) \otimes 2} \circ \Sigma_{q_{0}, q_{0}}\left(\frac{y}{n \gamma_{j}}\right)\right|^{2} \mathrm{~d}^{2 q_{0}} y .
\end{aligned}
$$

We now use the bound of the Dirichlet kernel given by Lemma A.3, the definition of $f$ given by Equation (1.2) with the boundedness of $f^{*}$, the bound of $\widehat{h}_{j}^{(K)}$ given by Equation (2.15). Then we deduce that

$$
\mathbb{E}\left[\left|S_{n, j}^{(0)}\right|^{2}\right] \leq C \gamma_{j}^{-2 q_{0}(1-2 d)} \gamma_{j}^{2(2 K+1)} I_{n, j}=C \gamma_{j}^{4\left(\delta\left(q_{0}\right)+K\right)} I_{n, j},
$$

where $\delta(\cdot)$ is defined by (2.1) and where for any $j, n$

$$
I_{n, j}=n^{-2 q_{0}(1-2 d)} \int_{\left(-n \gamma_{j} \pi, n \gamma_{j} \pi\right)^{2 q_{0}}} g \circ \Sigma_{q_{0}, q_{0}}\left(\frac{y}{n}\right)\left(\prod_{i=1}^{2 q_{0}}\left|y_{i}\right|^{-2 d}\right) \mathrm{d} y_{1} \cdots \mathrm{d} y_{2 q_{0}},
$$

with, for all $\left(\xi_{1}, \xi_{2}\right) \in \mathbb{R}^{2}$,

$$
g\left(\xi_{1}, \xi_{2}\right)=\left(1+\left|n\left\{\xi_{1}+\xi_{2}\right\}\right|\right)^{-2} \frac{\left|\gamma_{j}\left\{\xi_{1} / \gamma_{j}\right\}\right|^{2(M-K)}\left|\gamma_{j}\left\{\xi_{2} / \gamma_{j}\right\}\right|^{2(M-K)}}{\left[\left(1+\left|\gamma_{j}\left\{\xi_{1} / \gamma_{j}\right\}\right|\right)\left(1+\left|\gamma_{j}\left\{\xi_{2} / \gamma_{j}\right\}\right|\right)\right]^{2(M+\alpha)}} .
$$


We now bound the integral $I_{n, j}$. Observe that for any $y=\left(y_{1}, \cdots, y_{2 q_{0}}\right) \in\left(-n \gamma_{j} \pi, n \gamma_{j} \pi\right)^{2 q_{0}}$

$$
\left|y_{i}+\cdots+y_{q_{0}}\right| \leq n \gamma_{j}\left(q_{0}-i+1\right) \pi \quad \text { and } \quad\left|y_{q_{0}+i}+\cdots+y_{2 q_{0}}\right| \leq n \gamma_{j}\left(q_{0}-i+1\right) \pi .
$$

Thereafter, we set

$$
u_{1}=\frac{y_{1}+\cdots+y_{q_{0}}}{n}, \cdots, u_{q_{0}}=\frac{y_{q_{0}}}{n}, v_{1}=\frac{y_{q_{0}+1}+\cdots+y_{2 q_{0}}}{n}, \cdots, v_{q_{0}}=\frac{y_{2 q_{0}}}{n} .
$$

Then

$$
I_{n, j} \leq c_{0} \int_{u_{1}=-q_{0} \gamma_{j} \pi}^{q_{0} \gamma_{j} \pi} \int_{v_{1}=-q_{0} \gamma_{j} \pi}^{q_{0} \gamma_{j} \pi} g\left(u_{1}, v_{1}\right) J_{q_{0}, \gamma_{j} \pi}\left(u_{1} ; 2 d 1_{q_{0}}\right) J_{q_{0}, \gamma_{j} \pi}\left(v_{1} ; 2 d 1_{q_{0}}\right) \mathrm{d} u_{1} \mathrm{~d} v_{1},
$$

where we used the definition of $J_{m, a}(s ; \beta)$ in Lemma A.6 with the notation $1_{q_{0}}$ for the $q_{0^{-}}$ dimensional vector with all entries equal to 1 , that is, we set $m=q_{0}, a=\gamma_{j} \pi, \beta_{1}=\cdots=$ $\beta_{m}=2 d$ in (A.14). We now apply Lemma A.6. Since $q_{0}<1 /(1-2 d)$, we are in Case (i) of and we obtain

$$
J_{q_{0}, \gamma_{j} \pi}\left(z ; 2 d 1_{q_{0}}\right) \leq C|z|^{-2 \delta(m)}, \quad z \in \mathbb{R},
$$

for some constant $C>0$. This bound with the inequality $|\{u\}| \leq|u|$ and the expression of $g$ given by (7.13) yields

$$
I_{n, j} \leq C \int_{-q_{0} \gamma_{j} \pi}^{q_{0} \gamma_{j} \pi} \int_{-q_{0} \gamma_{j} \pi}^{q_{0} \gamma_{j} \pi} \frac{\left|\gamma_{j}\left\{u_{1} / \gamma_{j}\right\}\right|^{2\left(M-K-\delta\left(q_{0}\right)\right)}\left|\gamma_{j}\left\{v_{1} / \gamma_{j}\right\}\right|^{2\left(M-K-\delta\left(q_{0}\right)\right)} \mathrm{d} u_{1} \mathrm{~d} v_{1}}{\left(1+n\left|\left\{u_{1}+v_{1}\right\}\right|\right)^{2}\left[\left(1+\left|\gamma_{j}\left\{u_{1} / \gamma_{j}\right\}\right|\right)\left(1+\left|\gamma_{j}\left\{v_{1} / \gamma_{j}\right\}\right|\right)\right]^{2(M+\alpha)}} .
$$

By $2 \pi$-periodicity of $u \mapsto\{u\}$, we observe that the integrand is $\left(2 \pi \gamma_{j}\right)$-periodic with respect to both variables $u_{1}$ and $v_{1}$. Thus the integral on $\left(-q_{0} \gamma_{j} \pi, q_{0} \gamma_{j} \pi\right)^{2}$ equals $q_{0}^{2}$ times the integral on $\left(-\gamma_{j} \pi, \gamma_{j} \pi\right)^{2}$. We get that

$$
I_{n, j} \leq C \int_{u_{1}=-\gamma_{j} \pi}^{\gamma_{j} \pi} \int_{v_{1}=-\gamma_{j} \pi}^{\gamma_{j} \pi} \frac{\left|u_{1}\right|^{2\left(M-K-\delta\left(q_{0}\right)\right)}\left|v_{1}\right|^{2\left(M-K-\delta\left(q_{0}\right)\right)} \mathrm{d} u_{1} \mathrm{~d} v_{1}}{\left(1+n\left|\left\{u_{1}+v_{1}\right\}\right|\right)^{2}\left(1+\left|u_{1}\right|\right)^{2(M+\alpha)}\left(1+\left|v_{1}\right|\right)^{2(M+\alpha)}} .
$$

By assumption $2\left(M-K-\delta\left(q_{0}\right)\right) \geq 0$, then for any $t \in \mathbb{R}$,

$$
|t|^{2\left(M-K-\delta\left(q_{0}\right)\right)} \leq(1+|t|)^{2\left(M-K-\delta\left(q_{0}\right)\right)} \leq(1+|t|)^{2(M-K)} .
$$

It implies that

$$
I_{n, j} \leq C \int_{u_{1}=-\gamma_{j} \pi}^{\gamma_{j} \pi} \int_{v_{1}=-\gamma_{j} \pi}^{\gamma_{j} \pi} \frac{\mathrm{d} u_{1} \mathrm{~d} v_{1}}{\left(1+n\left|\left\{u_{1}+v_{1}\right\}\right|\right)^{2}\left(1+\left|u_{1}\right|\right)^{2(K+\alpha)}\left(1+\left|v_{1}\right|\right)^{2(K+\alpha)}} .
$$

We now apply Lemma A.7 with

$$
S=2(K+\alpha), \beta_{1}=\beta_{2}=0 .
$$

By assumption $S>1$. Then $I_{n, j} \leq C n^{-1}$ and the conclusion follows from (7.12).

\section{The leading term of the SCAlogram AND its ASYMPtotic Behavior}

Suppose $q_{0} \geq 2$. We will show that the leading term of $S_{n, j}$ is $S_{n, j}^{\left(q_{0}-1\right)}$ defined in (6.5). It is an element of the chaos of order $2 q_{0}-2\left(q_{0}-1\right)=2$ and after renormalization it will converge to a Rosenblatt random variable. We first study the asymptotic behavior of $S_{n, j}-S_{n, j}^{\left(q_{0}-1\right)}$ which is a sum of random variables in chaoses 4,6 up to $2 q_{0}$. We actually show in the next result that, under the normalization of $S_{n, j}^{\left(q_{0}-1\right)}$, this term is negligible. 
Corollary 8.1. Assume $q_{0} \geq 2$ and $M \geq \delta\left(q_{0}\right)+K$. Then, as $j, n \rightarrow \infty$,

$$
n^{1-2 d} \gamma_{j}^{-2\left(\delta\left(q_{0}\right)+K\right)}\left(\sum_{p=0}^{q_{0}-2} p !\left(\begin{array}{c}
q_{0} \\
p
\end{array}\right)^{2}\left\|S_{n, j}^{(p)}\right\|_{2}\right) \rightarrow 0,
$$

Proof. The limit (8.1) is a direct consequence of Propositions 7.1 and 7.2 , observing that $1-2 d=1-2 \delta(1)<1-2 \delta\left(q_{0}-p\right)$ for all $p=1,2, \ldots, q_{0}-2$ and that $\delta\left(q_{0}\right)>0$ and $q_{0} \geq 2$ imply $1-2 d<1 / 2$.

We consider the limit in distribution of the corresponding term $n^{1-2 d} \gamma_{j}^{-2\left(\delta\left(q_{0}\right)+K\right)} S_{n, j}^{\left(q_{0}-1\right)}$. With Corollary 8.1, this will provide the proof of Theorem 3.1 in the case $q_{0} \geq 2$. However, to cover the $m$-dimensional case with $m \geq 2$, we need to define a multivariate $S_{n, j}^{(p)}$ that will be denoted by $\mathbf{S}_{n, j}^{(p)}$. Let $0<p<q_{0}$. Define a $\mathbb{C}^{m}$-valued function $\widehat{\boldsymbol{\kappa}}_{j}^{(p)}$ by applying (6.8) component-wise with $h_{j}$ replaced by $h_{\ell, j}, \ell=1, \ldots, m$. Define a $\mathbb{C}^{m}$-valued function $\mathbf{g}_{p}$ by (6.6) with $\widehat{\kappa}_{j}^{(p)}$ replaced by $\widehat{\boldsymbol{\kappa}}_{j}^{(p)}$. Finally define $\mathbf{S}_{n, j}^{(p)}$ as a $m$-dimensional random vector defined by (6.5) with $g_{p}$ replaced by $\mathbf{g}_{p}$.

Proposition 8.1. Suppose that Assumptions $\boldsymbol{A}$ hold with $2 \leq q_{0}<1 /(1-2 d)$ and $M \geq K$. Then, for any diverging sequence $\left(n_{j}\right)$, as $j \rightarrow \infty$, we have

$$
n_{j}^{1-2 d} \gamma_{j}^{-2\left(\delta\left(q_{0}\right)+K\right)} \mathbf{S}_{n_{j}, j}^{\left(q_{0}-1\right)} \stackrel{\mathcal{L}}{\longrightarrow} f^{*}(0)^{q_{0}} \mathbf{L}_{q_{0}-1} Z_{d}(1) .
$$

where $Z_{d}(1)$ and $\mathbf{L}_{q_{0}-1}$ are the same as in Theorem 3.1.

Proof. Using (6.12) component-wise with $p=q_{0}-1$, observing that $2 q_{0}-2 p=2$ and making the change of variable $y=n \gamma_{j} \xi$ in the multiple stochastic integral, we get, using the selfsimilarity of the Wiener process,

$$
\begin{aligned}
\mathbf{S}_{n, j}^{\left(q_{0}-1\right)} & =\widehat{I}_{2}\left(D_{n} \circ \Sigma_{2}\left(\gamma_{j} \times \cdot\right) \times\left[\sqrt{f} \mathbb{1}_{(-\pi, \pi)}\right]^{\otimes 2} \times \widehat{\boldsymbol{\kappa}}_{j}^{\left(q_{0}-1\right)}\right) \\
& \stackrel{d}{=} \frac{1}{n \gamma_{j}} \widehat{I}_{2}\left(D_{n} \circ \Sigma_{2}\left(n^{-1} \times \cdot\right) \times \mathbb{1}_{\left(-\gamma_{j} \pi, \gamma_{j} \pi\right)}^{\otimes 2}\left(n^{-1} \times \cdot\right) \times \mathbf{f}_{j}\right),
\end{aligned}
$$

where, for all $\xi \in \mathbb{R}^{2}$,

$$
\mathbf{f}_{j}\left(n \gamma_{j} \xi\right)=\sqrt{f}^{\otimes 2}(\xi) \times \widehat{\boldsymbol{\kappa}}_{j}^{\left(q_{0}-1\right)}(\xi) .
$$

Here $\stackrel{d}{=}$ means that the two vectors have same distributions for all $n, j \geq 1$. We will use Lemma A.3 which involves fractional parts. Let us express $1_{\left(-\gamma_{j} \pi, \gamma_{j} \pi\right)}^{\otimes 2}$ as a sum of indicator functions on the following pairwise disjoint domains,

$$
\Gamma_{j}^{(s)}=\left\{t=\left(t_{1}, t_{2}\right) \in\left(-\gamma_{j} \pi, \gamma_{j} \pi\right)^{2},\left|t_{1}+t_{2}-2 \pi s\right|<\pi\right\}, \quad s \in \mathbb{Z} .
$$

Hence we obtain

$$
\begin{gathered}
\mathbf{S}_{n, j}^{\left(q_{0}-1\right)} \stackrel{d}{=} \frac{1}{n \gamma_{j}} \sum_{s \in \mathbb{Z}} \mathbf{I}_{n, j}^{(s)} . \\
\mathbf{I}_{n, j}^{(s)}=\widehat{I}_{2}\left(D_{n} \circ \Sigma_{2}\left(n^{-1} \times \cdot\right) \times \mathbb{1}_{\Gamma_{j}^{(s)}}\left(n^{-1} \times \cdot\right) \times \mathbf{f}_{j}\right) .
\end{gathered}
$$

Proposition 8.1 follows from the following three convergence results, valid for all fixed $m \in \mathbb{Z}$. 
(a) If $s=0$, then, as $j \rightarrow \infty$,

$$
\left(n_{j} \gamma_{j}\right)^{-2 d} \gamma_{j}^{-2\left(\delta\left(q_{0}-1\right)+K\right)} \mathbf{I}_{n_{j}, j}^{(0)} \stackrel{L^{2}(\Omega)}{\longrightarrow}\left(f^{*}(0)\right)^{q_{0}} \mathbf{L}_{q_{0}-1} Z_{d}(1) .
$$

(b) We have, as $j \rightarrow \infty$,

$$
\sup _{s \neq 0} \mathbb{E}\left[\left(n_{j} \gamma_{j}\right)^{-4 d} \gamma_{j}^{-4\left(\delta\left(q_{0}-1\right)+K\right)}\left|\mathbf{I}_{n_{j}, j}^{(s)}\right|^{2}\right] \rightarrow 0
$$

(c) We have, as $j \rightarrow \infty$,

$$
\sum_{s \notin \gamma_{j} \mathbb{Z}} \mathbb{E}\left[\left(n_{j} \gamma_{j}\right)^{-4 d} \gamma_{j}^{-4\left(\delta\left(q_{0}-1\right)+K\right)}\left|\mathbf{I}_{n_{j}, j}^{(s)}\right|^{2}\right] \rightarrow 0
$$

To show that this is sufficient to prove the proposition, observe that, for any $t=\left(t_{1}, t_{2}\right) \in \Gamma_{j}^{(s)}$, we have

$$
2 \pi|s|-\pi<2 \pi|s|-\left|t_{1}+t_{2}-2 \pi s\right| \leq\left|t_{1}+t_{2}\right|<2 \gamma_{j} \pi
$$

Hence the domain $\Gamma_{j}^{(s)}$ is empty if $|s|>\gamma_{j}+1 / 2$. We use (8.8) for the two values $s=\gamma_{j}$ and $s=-\gamma_{j}$ and (8.9) for the values $s \notin \gamma_{j} \mathbb{Z}$. Thus (8.8) and (8.9) imply

$$
\left(n \gamma_{j}\right)^{-2 d} \gamma_{j}^{-2\left(\delta\left(q_{0}-1\right)+K\right)} \sum_{s \neq 0} \mathbf{I}_{n, j}^{(s)} \stackrel{L^{2}(\Omega)}{\longrightarrow} 0
$$

Observe also that the normalizing factor in the left-hand side of (8.2) can be written as

$$
n^{1-2 d} \gamma_{j}^{-2\left(\delta\left(q_{0}\right)+K\right)}=\left(n \gamma_{j}\right)\left(\left(n \gamma_{j}\right)^{-2 d} \gamma_{j}^{-2\left(\delta\left(q_{0}-1\right)+K\right)}\right),
$$

by using the definition of $\delta$ in (2.1). The last two displays, (8.5) and (8.7) yield (8.2).

It only remains to prove (8.7), (8.8) and (8.9).

a) We first show (8.7). Since $\mathbf{I}_{n, j}^{(0)}$ and $Z_{d}(1)$ are defined as stochastic integrals of order 2 , (8.7) is equivalent to the $L^{2}\left(\mathbb{R}^{2}\right)$ convergence of the normalized corresponding kernels. We show the latter by a dominated convergence argument. These kernels are given in (8.6) and (3.3) respectively. Observe that, as $n \rightarrow \infty, D_{n}(\theta / n) \rightarrow\left(\mathrm{e}^{\mathrm{i} \theta}-1\right) /(i \theta)$ by (6.7), for all $y \in \mathbb{R}^{2}$,

$$
D_{n}\left(n^{-1}\left(y_{1}+y_{2}\right)\right) \rightarrow \frac{\exp \left(\mathrm{i}\left(y_{1}+y_{2}\right)\right)-1}{\left(\mathrm{i}\left(y_{1}+y_{2}\right)\right)} .
$$

By (1.1), we have, as $\left(n \gamma_{j}\right) \rightarrow \infty$, for all $y \in \mathbb{R}^{2}$,

$$
\sqrt{f}^{\otimes 2}\left(y /\left(n \gamma_{j}\right)\right) \sim f^{*}(0)\left(n \gamma_{j}\right)^{2 d}\left|y_{1}\right|^{-d}\left|y_{2}\right|^{-d} .
$$

Now applying Lemma A.2 to the $m$ entries of $\widehat{\boldsymbol{\kappa}}_{j}^{(p)}$ with $p=q_{0}-1$, we get that, as $j \rightarrow \infty$, for all $y \in \mathbb{R}^{2}$,

$$
\gamma_{j}^{\left(q_{0}-1\right)(1-2 d)-(2 K+1)} \widehat{\boldsymbol{\kappa}}_{j}^{\left(q_{0}-1\right)}\left(y /\left(n_{j} \gamma_{j}\right)\right) \rightarrow\left(f^{*}(0)\right)^{q_{0}-1} \mathbf{L}_{q_{0}-1} .
$$

The last three convergences and $2 \delta\left(q_{0}-1\right)=1-\left(q_{0}-1\right)(1-2 d)$ yield the pointwise convergence of the normalized kernels defining the stochastic integrals appearing in the left-hand side of (8.7) towards the kernel of the right-hand side. 
It remains to bound these kernels by an $L^{2}\left(\mathbb{R}^{2}\right)$ function not depending on $j, n$. We may take $m=1$ without loss of generality for this purpose, since component-wise bounds are sufficient. If $y / n \in \Gamma_{j}^{(0)}$, we have, by Lemma A.3,

$$
\left|D_{n}\left(\left(y_{1}+y_{2}\right) / n\right)\right| \leq C\left(1+\left|y_{1}+y_{2}\right|\right)^{-1},
$$

for some constant $C>0$. By (1.1), since $f^{*}$ is bounded, we have, for all $y=\left(y_{1}, y_{2}\right) \in$ $\left(-n \gamma_{j} \pi, n \gamma_{j} \pi\right)$

$$
\left|\left(n \gamma_{j}\right)^{-2 d} \sqrt{f}^{\otimes 2}\left(y /\left(n \gamma_{j}\right)\right)\right| \leq C\left|y_{1}\right|^{-d}\left|y_{2}\right|^{-d},
$$

where $C$ is a constant. Since $q_{0}-1<1 /(1-2 d)$, Lemma A.1 implies that, for all $\zeta \in \mathbb{R}^{2}$ and some constant $C$,

$$
\left|\gamma_{j}^{-2\left(\delta\left(q_{0}-1\right)+K\right)} \widehat{\kappa}_{j}^{\left(q_{0}-1\right)}(\zeta)\right| \leq C .
$$

The bounds (8.10), (8.11) and (8.12) imply that $\left(n \gamma_{j}\right)^{-2 d} \gamma_{j}^{-2\left(\delta\left(q_{0}-1\right)+K\right)} I_{n, j}^{(0)}=\widehat{I}_{2}(g)$ with

$$
|g(y)|^{2} \leq C\left(1+\left|y_{1}+y_{2}\right|\right)^{-2}\left|y_{1}\right|^{-2 d}\left|y_{2}\right|^{-2 d}, \quad y=\left(y_{1}, y_{2}\right) \in \mathbb{R}^{2},
$$

for some positive constant $C$. Since we assumed $2<1 /(1-2 d)$. Then, applying Lemma A.5 with $M_{1}=2, q=2$, and $a=0$, we obtain that this function is integrable and the convergence (8.7) follows.

b) Let us now prove (8.8). Again we may take $m=1$ without loss of generality since the bound can be applied component-wise to derive the case $m \geq 2$. Observe that the bounds (8.11) and (8.12) can be used for $y / n \in \Gamma_{j}^{(s)}$, while the bound (8.10) becomes

$$
\left|D_{n}\left(\left(y_{1}+y_{2}\right) / n\right)\right|^{2} \leq C\left(1+\left|y_{1}+y_{2}-2 \pi n s\right|\right)^{-2},
$$

Hence in this case, we obtain that $\left(n \gamma_{j}\right)^{-2 d} \gamma_{j}^{-2\left(\delta\left(q_{0}-1\right)+K\right)} I_{n, j}^{(s)}=\widehat{I}_{2}(g)$ with

$$
|g(y)|^{2} \leq C\left(1+\left|y_{1}+y_{2}-2 \pi n s\right|\right)^{-2}\left|y_{1}\right|^{-2 d}\left|y_{2}\right|^{-2 d}, \quad y=\left(y_{1}, y_{2}\right) \in \mathbb{R}^{2},
$$

for some positive constant $C$. Using the assumption $2<1 /(1-2 d)$, from Lemma A.5 applied with $q=2, a=2 \pi n s$ and $M_{1}=2$, we get (8.8).

c) Finally we prove (8.9) with $m=1$. We need to further partition $\Gamma_{j}^{s}$ into

$$
\Gamma_{j}^{(s, \sigma)}=\left\{t \in \Gamma_{j}^{s}, t / \gamma_{j}-2 \pi \sigma \in(-\pi, \pi)^{2}\right\}, \quad \sigma \in \mathbb{Z}^{2} .
$$

Note that for all $t=\left(t_{1}, t_{2}\right) \in \Gamma_{j}^{(s, \sigma)}$, we have, for any $i=1,2$,

$$
\left|2 \pi \sigma_{i}\right| \leq\left|t_{i} / \gamma_{j}-2 \pi \sigma_{i}\right|+\left|t_{i} / \gamma_{j}\right|<2 \pi .
$$

Hence $\Gamma_{j}^{(s, \sigma)}=\emptyset$ for all $\sigma$ out of the integer rectangle $R=\{-1,0,1\}^{2}$. Then we obtain

$$
\left(n \gamma_{j}\right)^{-2 d} \gamma_{j}^{-2\left(\delta\left(q_{0}-1\right)+K\right)} I_{n, j}^{(s)}=\sum_{\sigma \in R} \widehat{I}_{2}\left(g_{\sigma}^{(s)}\right)
$$

where, for all $y \in \mathbb{R}^{2}$,

$$
g_{\sigma}^{(s)}(y)=\left(n \gamma_{j}\right)^{-2 d} \gamma_{j}^{-2\left(\delta\left(q_{0}-1\right)+K\right)} D_{n} \circ \Sigma_{2}(y / n) \times \mathbb{1}_{\Gamma_{j}^{(s, \sigma)}}(y / n) \times f_{j}(y) .
$$

Since $R$ is a finite set, to obtain the limit (8.9), it is sufficient to show that, for any fixed $\sigma \in R$, as $j, n \rightarrow \infty$,

$$
\sum_{s \notin \gamma_{j} \mathbb{Z}} \int\left|g_{\sigma}^{(s)}(y)\right|^{2} \mathrm{~d}^{2} y \rightarrow 0 \text {. }
$$


For $\zeta \in 2 \pi \sigma+(-\pi, \pi)^{2}$, we use a sharper bound than (8.12), namely, by Lemma A.1,

$$
\left|\gamma_{j}^{-2\left(\delta\left(q_{0}-1\right)+K\right)} \widehat{\kappa}_{j}^{\left(q_{0}-1\right)}(\zeta)\right|^{2} \leq C k_{j}^{\otimes 2}(\zeta-2 \pi \sigma) \quad \text { where } \quad k_{j}(u)=\left(1+\gamma_{j}|u|\right)^{-2 \delta\left(q_{0}-1\right)} .
$$

With (8.11) and (8.13), it follows that

$$
\left|g_{\sigma}^{(s)}(y)\right|^{2} \leq C \frac{k_{j}^{\otimes 2}\left(y /\left(n \gamma_{j}\right)-2 \pi \sigma\right)}{\left(1+\left|y_{1}+y_{2}-2 \pi n s\right|\right)^{2}}\left|y_{1}\right|^{-2 d}\left|y_{2}\right|^{-2 d}, \quad y=\left(y_{1}, y_{2}\right) \in \mathbb{R}^{2} .
$$

Let us set $w=\left(w_{1}, w_{2}\right)$ with $w_{1}=y_{1} /\left(n \gamma_{j}\right)-2 \pi \sigma_{1}$ and $w_{2}=y_{2} /\left(n \gamma_{j}\right)-2 \pi \sigma_{2}$. Using the bound (8.17) and that $y / n \in \Gamma_{j}^{(s, \sigma)}$ implies $w \in \Delta_{j}^{(s, \sigma)}$ with

$$
\Delta_{j}^{(s, \sigma)}=\left\{\left(w_{1}, w_{2}\right) \in(-\pi, \pi)^{2},\left|\gamma_{j}\left(w_{1}+w_{2}\right)-2 \pi\left(s-\gamma_{j}\left(\sigma_{1}+\sigma_{2}\right)\right)\right|<\pi\right\},
$$

we get

$$
\int\left|g_{\sigma}^{(s)}(y)\right|^{2} \mathrm{~d}^{2} y \leq C\left(n \gamma_{j}\right)^{2(1-2 d)} \int_{\Delta_{j}^{(s, \sigma)}} \frac{k_{j}^{\otimes 2}(w)\left|w_{1}+2 \pi \sigma_{1}\right|^{-2 d}\left|w_{2}+2 \pi \sigma_{2}\right|^{-2 d}}{\left(1+n\left|\gamma_{j}\left(w_{1}+w_{2}\right)-2 \pi\left(s-\gamma_{j}\left(\sigma_{1}+\sigma_{2}\right)\right)\right|\right)^{2}} \mathrm{~d}^{2} w,
$$

Since $\left|w_{i} \pm 2 \pi\right|>\pi>\left|w_{i}\right|$ for $w \in \Delta_{j}^{(s, \sigma)}$, we have for $\sigma \in R$,

$$
\int\left|g_{\sigma}^{(s)}(y)\right|^{2} \mathrm{~d}^{2} y \leq C\left(n \gamma_{j}\right)^{2(1-2 d)} \int_{\Delta_{j}^{(s, \sigma)}} \frac{k_{j}^{\otimes 2}(w)\left|w_{1}\right|^{-2 d}\left|w_{2}\right|^{-2 d}}{\left(1+n\left|\gamma_{j}\left(w_{1}+w_{2}\right)-2 \pi\left(s-\gamma_{j}\left(\sigma_{1}+\sigma_{2}\right)\right)\right|\right)^{2}} \mathrm{~d}^{2} w .
$$

We shall apply Lemma A.5 after having conveniently bounded $k_{j}$ in the numerator of the previous ratio. Let $\beta<1$ to be set later arbitrarily close to 1 . Since $2 \delta\left(q_{0}-1\right) \geq \beta-2 d+2 \delta\left(q_{0}\right)$, we have

$$
\begin{aligned}
k_{j}(u) & =\left(1+\gamma_{j}|u|\right)^{-2 \delta\left(q_{0}-1\right)} \\
& \leq\left(1+\gamma_{j}|u|\right)^{2 d-\beta}\left(1+\gamma_{j}|u|\right)^{-2 \delta\left(q_{0}\right)} .
\end{aligned}
$$

Observe that, for all $w \in \Delta_{j}^{(s, \sigma)}$ we have

$$
\gamma_{j}\left(\left|w_{1}\right| \vee\left|w_{2}\right|\right) \geq \gamma_{j}\left|\left(w_{1}+w_{2}\right) / 2\right| \geq \pi\left(\left|s-\gamma_{j}\left(\sigma_{1}+\sigma_{2}\right)\right|-1 / 2\right) \geq \pi\left|s-\gamma_{j}\left(\sigma_{1}+\sigma_{2}\right)\right| / 2 .
$$

In the last inequality, we used that $s \notin \gamma_{j} \mathbb{Z}$ and that $s, \gamma_{j}, \sigma_{1}$ and $\sigma_{2}$ are integers so that $\left|s-\gamma_{j}\left(\sigma_{1}+\sigma_{2}\right)\right| \geq 1$.

Using $0<q_{0}<1 /(1-2 d)$, we have $2 \delta\left(q_{0}\right)>0$, and, choosing $\beta$ close enough to 1 , we have $\beta-2 d>0$. Hence, the last two displays yield, for all $w \in \Delta_{j}^{(s, \sigma)}$ with $s \notin \gamma_{j} \mathbb{Z}$,

$$
k_{j}^{\otimes 2}(w) \leq\left|\gamma_{j} w_{1}\right|^{2 d-\beta}\left|\gamma_{j} w_{2}\right|^{2 d-\beta}\left(1+\pi\left|s-\gamma_{j}\left(\sigma_{1}+\sigma_{2}\right)\right| / 2\right)^{-2 \delta\left(q_{0}\right)} .
$$

Inserting this bound in (8.18) and setting $t=n \gamma_{j} w$, we obtain

$$
\begin{aligned}
& \int\left|g_{\sigma}^{(s)}(y)\right|^{2} \mathrm{~d}^{2} y \\
& \leq C \frac{n^{-4 d+2 \beta}}{\left|s-\gamma_{j}\left(\sigma_{1}+\sigma_{2}\right)\right|^{2 \delta\left(q_{0}\right)}} \int_{\mathbb{R}^{2}} \frac{\left|t_{1} t_{2}\right|^{-\beta}}{\left(1+\left|t_{1}+t_{2}-2 \pi n\left(s-\gamma_{j}\left(\sigma_{1}+\sigma_{2}\right)\right)\right|\right)^{2}} \mathrm{~d}^{2} t .
\end{aligned}
$$

For $\beta$ close enough to 1 , we may apply Lemma A.5 with $q=2, d=\beta / 2, M_{1}=2$ and $a=2 \pi n\left(s-\gamma_{j}\left(\sigma_{1}+\sigma_{2}\right)\right)$ to bound the previous integral. Using again that $s \notin \gamma_{j} \mathbb{Z}$ and that 
$s, \gamma_{j}, \sigma_{1}$ and $\sigma_{2}$ are integers, we have $|a| \geq 2 \pi n$ and thus $1+|a| \asymp|a|$. We get, for all $s \notin \gamma_{j} \mathbb{Z}$

$$
\int\left|g_{\sigma}^{(s)}(y)\right|^{2} \mathrm{~d}^{2} y \leq C n^{1-4 d}\left|s-\gamma_{j}\left(\sigma_{1}+\sigma_{2}\right)\right|^{1-2 \delta\left(q_{0}\right)-2 \beta}
$$

where $C$ is some positive constant.

Now choose $\beta$ close enough to 1 so that $2 \delta\left(q_{0}\right)+2 \beta-1>1$. It follows that

$$
\sum_{k \neq 0}|k|^{1-2 \delta\left(q_{0}\right)-2 \beta}<\infty
$$

Since our assumptions imply $d>1 / 4$, the last two displays imply (8.15) and the proof is finished.

\section{Proof of the MAin Results}

Proof Theorem 3.1. We first prove the result in Casea, In this case $q_{0}=1$ and thus $H_{q_{0}}\left(X_{t}\right)=$ $X_{t}$. Let $(v(s))_{s \in \mathbb{Z}}$ be the Fourier coefficients of $\sqrt{2 \pi f}$, so that the convergence

$$
\sqrt{2 \pi f(\lambda)}=\widehat{v}(\lambda)=\sum_{s \in \mathbb{Z}} v(s) \mathrm{e}^{-\mathrm{i} \lambda s}
$$

holds in $L^{2}(-\pi, \pi)$. It follows that $\left\{X_{t}\right\}_{t \in \mathbb{Z}}$ can be represented as

$$
X_{t}=\sum_{s \in \mathbb{Z}} v(t-s) \xi_{s}, \quad t \in \mathbb{Z},
$$

where $\left\{\xi_{t}\right\}_{t \in \mathbb{Z}}$ is an i.i.d. sequence of standard Gaussian r.v.'s. Applying (2.13) with $H_{q_{0}}\left(X_{t}\right)=$ $X_{t}$ we obtain that

$$
\mathbf{W}_{j, k}=\gamma_{j}^{d+K}\left[\begin{array}{c}
Z_{1, j, k} \\
\vdots \\
Z_{m, j, k}
\end{array}\right]
$$

where

$$
Z_{\ell, j, k}=\sum_{t \in \mathbb{Z}} v_{\ell, j}\left(\gamma_{j} k-t\right) \xi_{t}
$$

with

$$
v_{\ell, j}(u)=\gamma_{j}^{-d-K} \sum_{s \in \mathbb{Z}} h_{\ell, j}^{(K)}(u-s) v(s), \quad u \in \mathbb{Z} .
$$

Hence

$$
\widehat{v}_{\ell, j}(\lambda)=\gamma_{j}^{-d-K} \widehat{h}_{\ell, j}^{(K)}(\lambda) \widehat{v}(\lambda)=\gamma_{j}^{-d-K} \sqrt{2 \pi f(\lambda)} \widehat{h}_{\ell, j}^{(K)}(\lambda), \quad \lambda \in(-\pi, \pi) .
$$

Observe that (1.1), (2.8) and (2.14) imply, for some positive constant $C$,

$$
\left|\widehat{v}_{\ell, j}(\lambda)\right| \leq C \gamma_{j}^{1 / 2} \frac{\left|\gamma_{j} \lambda\right|^{M-(K+d)}}{\left(1+\gamma_{j}|\lambda|\right)^{\alpha+M}}, \quad \lambda \in(-\pi, \pi) .
$$

On the other hand, (1.1), (2.10) and (2.14) imply

$$
\lim _{j \rightarrow+\infty} \gamma_{j}^{-1 / 2} \widehat{v}_{\ell, j}\left(\gamma_{j}^{-1} \lambda\right) \mathrm{e}^{\mathrm{i} \Phi_{j}(\lambda)}=\sqrt{2 \pi f^{*}(0)}|\lambda|^{-(K+d)} \widehat{h}_{\ell, \infty}(\lambda), \quad \lambda \in \mathbb{R}, \ell=1, \ldots, m .
$$


Thus, if $M \geq K+d$, Assumptions A imply Condition B in [30] with $N=m, \delta=\alpha+K+d$, $\lambda_{i, j}=\lambda_{i, \infty}=0, \Phi_{i, j}=\Phi_{j}, v_{i, j}^{*}=(2 \pi)^{-1 / 2} \widehat{v}_{i, j}$ and $v_{i, \infty}^{*}(\lambda)=\sqrt{f^{*}(0)}|\lambda|^{-(K+d)} \widehat{h}_{i, \infty}(\lambda)$ for $i=1, \ldots, N$ and $j \geq 1$. Moreover we may apply Theorem 1 in [30] and obtain, as $j \rightarrow \infty$,

$$
n_{j}^{-1 / 2} \sum_{k=0}^{n_{j}-1}\left[\begin{array}{c}
Z_{1, j, k}^{2}-\mathbb{E}\left[Z_{1, j, k}^{2}\right] \\
\vdots \\
Z_{N, j, k}^{2}-\mathbb{E}\left[Z_{N, j, k}^{2}\right]
\end{array}\right] \stackrel{\mathcal{L}}{\longrightarrow} \mathcal{N}(0, \Gamma)
$$

where $\Gamma$ is the $m \times m$ covariance matrix defined by (3.5). Since, by (3.1) and (9.1) $), n_{j}^{1 / 2} \gamma_{j}^{-2(d+K)} \overline{\mathbf{S}}_{n_{j}, j}$ is the left-hand side of the last display, we get (3.4).

We now consider Case b. Applying the basic decomposition (6.2) to each entries of $\overline{\mathbf{S}}_{n, j}$, Corollary 8.1 and Proposition 8.1 show that the leading term is obtained for $p=q_{0}-1$. Moreover the latter proposition specifies the limit.

Proof of Theorem 4.1. We first prove (4.5). Applying (4.4), (B.7) (with $h_{j}$ replaced by $g_{j}$ ) and the isometry property (B.5), we have

$$
\sigma_{j}^{2}=q_{0} ! \int_{(-\pi, \pi)^{q_{0}}} \frac{\left|\widehat{g}_{j} \circ \Sigma_{q_{0}}(\xi)\right|^{2}}{\left|1-\mathrm{e}^{-\mathrm{i} \Sigma_{q_{0}}(\xi)}\right|^{2 K}} f^{\otimes q_{0}}(\xi) \mathrm{d}^{q_{0}} \xi .
$$

Setting $\xi=2^{-j} \lambda$, we get

$$
\sigma_{j}^{2}=q_{0} ! 2^{-j\left(q_{0}-1\right)} \int_{\left(-2^{j} \pi, 2^{j} \pi\right)^{q_{0}}} \frac{\left|2^{-j / 2} \widehat{g}_{j} \circ \Sigma_{q_{0}}\left(2^{-j} \lambda\right)\right|^{2}}{\left|1-\mathrm{e}^{-\mathrm{i} \Sigma_{q_{0}}\left(2^{-j} \lambda\right)}\right|^{2 K}} f^{\otimes q_{0}}\left(2^{-j} \lambda\right) \mathrm{d}^{q_{0}} \lambda .
$$

Using Assumption (W-b) on $g_{j}$, and Condition (1.2) with $f^{*}$ bounded, the integrand is bounded, up to a multiplicative constant, by

$$
2^{2 j\left(K+d q_{0}\right)}\left(1+\left|\Sigma_{q_{0}}(\lambda)\right|\right)^{-2(\alpha+K)} \prod_{i=1}^{q_{0}}\left|\lambda_{i}\right|^{-2 d}
$$

since $(|x| /(1+|x|))^{M} \leq(|x| /(1+|x|))^{K}$ and $\left|1-\mathrm{e}^{-\mathrm{i} x}\right| \asymp|x|$. The displayed bound is integrable by Lemma A.5 with $M_{1}=2(\alpha+K), q=q_{0}$ and $a=0$. By dominated convergence, Assumption (W-C) on $\left(g_{j}\right)$ and continuity of $f^{*}$ at zero, we get, as $j \rightarrow \infty$,

$$
2^{-2 j\left(K+d q_{0}-\left(q_{0}-1\right) / 2\right)} \sigma_{j}^{2} \rightarrow q_{0} !\left(f^{*}(0)\right)^{q_{0}} \int_{\mathbb{R}^{q_{0}}} \frac{\left|\widehat{g}_{\infty} \circ \Sigma_{q_{0}}(\lambda)\right|^{2}}{\left|\Sigma_{q_{0}}(\lambda)\right|^{2 K}} \prod_{i=1}^{q_{0}}\left|\lambda_{i}\right|^{-2 d} \mathrm{~d}^{q_{0}} \lambda .
$$

Using (2.1) and the definition $L_{q_{0}}\left(\widehat{g}_{\infty}\right)$ in (3.7), we obtain (4.5).

To prove the convergence of the scalogram, we shall apply Theorem 3.1(b) with a sequence of multivariate filters $\left(\mathbf{h}_{j}\right)_{j \geq 0}$. To illustrate how this is done, suppose, for example, that we want to study the joint behavior of $W_{j-u, k}$ for $u \in\{0,1\}$. Recall that $j-1$ is a finer scale than $j$. Following the framework of [31], we consider the multivariate coefficients $\mathbf{W}_{j, k}=\left(W_{j, k}, W_{j-1,2 k}, W_{j-1,2 k+1}\right)$, since, in addition to the wavelet coefficients $W_{j, k}$ at scale $j$, there are twice as many wavelet coefficients $W_{j-1,2 k}, W_{j-1,2 k+1}$ at scale $j-1$. These coefficients can be viewed as the output of a multidimensional filter $\mathbf{h}_{j}$ defined as $\mathbf{h}_{j}(\tau)=\left(h_{j}(\tau), h_{j-1}(\tau), h_{j-1}\left(\tau+2^{j-1}\right)\right)$. These three entries correspond to $(u, v)$ equal to $(0,0),(1,0)$ and $(1,1)$, respectively, in the general case below. 
In the general case, each $\mathbf{h}_{j}$ is defined as follows. For all, $j \geq 0, u \in\{0, \ldots, j\}$ and $v \in\left\{0, \ldots, 2^{u}-1\right\}$, let $\ell=2^{u}+v$ and define a filter $h_{\ell, j}$ by

$$
h_{\ell, j}(t)=g_{j-u}\left(t+2^{j-u} v\right), \quad t \in \mathbb{Z} .
$$

Applying this definition and (4.1) with $\gamma_{j}=2^{j}$, we get

$$
W_{j-u, 2^{u} k+v}=\sum_{t \in \mathbb{Z}} h_{\ell, j}\left(2^{j} k-t\right) Y_{t} .
$$

These coefficients are stored in a vector $\mathbf{W}_{j, k}=\left[W_{\ell, j, k}\right]_{\ell}$, say of length $m=2^{p}-1$,

$$
W_{\ell, j, k}=W_{j-u, 2^{u} k+v}, \quad \ell=2^{u}+v=1,2, \ldots, m,
$$

which corresponds to the multivariate wavelet coefficient (2.7) with $\mathbf{h}_{j}(t)$ having components $h_{\ell, j}(t), \ell=1,2, \ldots, m$ defined by (9.2). This way of proceeding allows us to express the vector $\left[\widehat{\sigma}_{j-u}^{2}-\sigma_{j-u}^{2}\right]_{u=0, \ldots, p-1}$ as a linear function, up to a negligible remainder, of the vector $\overline{\mathbf{S}}_{n_{j}, j}$ defined by (3.1). Indeed observe that (4.2) implies, for any fixed $u$

$$
n_{j-u}=2^{u} n_{j}+O(1) .
$$

Hence (4.3) and (4.4) imply, for any fixed $u$,

$$
\begin{aligned}
\widehat{\sigma}_{j-u}^{2}-\sigma_{j-u}^{2} & =\frac{1}{n_{j-u}} \sum_{k=0}^{n_{j-u}}\left(W_{j-u, k}^{2}-\mathbb{E}\left[W_{j-u, k}^{2}\right]\right) \\
& =\frac{1}{n_{j-u}} \sum_{k=0}^{2^{u} n_{j}-1}\left(W_{j-u, k}^{2}-\mathbb{E}\left[W_{j-u, k}^{2}\right]\right)+O_{P}\left(\sigma_{j-u}^{2} / n_{j-u}\right), \quad j \geq u .
\end{aligned}
$$

Expanding $\sum_{k=0}^{2^{u} n_{j}-1}$ as $\sum_{v=0}^{2^{u}-1} \sum_{k^{\prime}=0}^{n_{j}-1}$ with $k=k^{\prime} 2^{u}+v$ and applying (9.3) and the last display, we obtain, for all $j \geq p$,

$$
\begin{aligned}
\widehat{\sigma}_{j-u}^{2}-\sigma_{j-u}^{2} & =\frac{1}{n_{j-u}} \sum_{v=0}^{2^{u}-1} \sum_{k^{\prime}=0}^{n_{j}-1}\left(W_{2^{u}+v, j, k}^{2}-\mathbb{E}\left[W_{2^{u}+v, j, k}^{2}\right]\right)+O_{P}\left(\sigma_{j-u}^{2} / n_{j-u}\right) \\
& =\frac{n_{j}}{n_{j-u}} \sum_{v=0}^{2^{u}-1} \bar{S}_{n_{j}, j}\left(2^{u}+v\right)+O_{P}\left(\sigma_{j-u}^{2} / n_{j-u}\right), \quad u=0, \ldots, p-1,
\end{aligned}
$$

where we denoted the entries of $\overline{\mathbf{S}}_{n_{j}, j}$ in (3.1) as $\left[\bar{S}_{n_{j}, j}(\ell)\right]_{\ell=1, \ldots, m}$.

Let us now check that $\left(\mathbf{h}_{j}\right)$ satisfies the assumptions of Theorem 3.1. By hypothesis $\left\{g_{j}\right\}$ verifies Assumptions (W-a $)-(\mathrm{W}-\mathrm{c})$. Hence, by (9.2), $\left\{\mathbf{h}_{j}\right\}$ satisfies (W-或). We further have that, for $\ell=2^{u}+v$ with $u \in\{0, \ldots, p-1\}$ and $v \in\left\{0, \ldots, 2^{u}-1\right\}$,

$$
\widehat{h}_{\ell, j}(\lambda)=\widehat{g}_{j-u}(\lambda) \mathrm{e}^{\mathrm{i} 2^{j-u} v \lambda}, \lambda \in(-\pi, \pi) .
$$

Hence (W-b) follows from the assumption on $g_{j}$. Using that $\gamma_{j}=2^{j}$, Condition (W-C) also follows with $\Phi_{j} \equiv 0$ and

$$
\widehat{h}_{\ell, \infty}(\lambda)=2^{-u / 2} \widehat{g}_{\infty}\left(2^{-u} \lambda\right) \mathrm{e}^{\mathrm{i} 2^{-u} v \lambda} .
$$

We can thus apply Theorem 3.1 and obtain (3.6), that is,

$$
n_{j}^{1-2 d} 2^{-2 j\left(\delta\left(q_{0}\right)+K\right)} \overline{\mathbf{S}}_{n_{j}, j} \stackrel{\mathcal{L}}{\longrightarrow} f^{*}(0)^{q_{0}} \mathbf{L}_{q_{0}-1} Z_{d}(1) .
$$


with $\mathbf{L}_{q_{0}-1}=\left[L_{q_{0}-1}\left(\widehat{h}_{\ell, \infty}\right)\right]_{\ell=1, \ldots, m}$. By (9.6) and (3.7), it turns out that, for $\ell=2^{u}+v$ with $u \in\{0, \ldots, p-1\}$ and $v \in\left\{0, \ldots, 2^{u}-1\right\}$,

$$
\begin{aligned}
L_{q_{0}-1}\left(\widehat{h}_{\ell, \infty}\right) & =\int_{\mathbb{R}^{q_{0}-1}} \frac{\left|2^{-u / 2} \widehat{g}_{\infty}\left(2^{-u}\left(t_{1}+\cdots+t_{p}\right)\right)\right|^{2}}{\left|u_{1}+\cdots+u_{q_{0}-1}\right|^{2 K}} \prod_{i=1}^{q_{0}-1}\left|t_{i}\right|^{-2 d} \mathrm{~d} t_{1} \cdots \mathrm{d} t_{q_{0}-1} \\
& =2^{-u-2 K u-2 d\left(q_{0}-1\right) u+u\left(q_{0}-1\right)} L_{q_{0}-1}\left(\widehat{g}_{\infty}\right) \\
& =2^{-2 u\left(\delta\left(q_{0}\right)+K\right)+u(2 d-1)} L_{q_{0}-1}\left(\widehat{g}_{\infty}\right)
\end{aligned}
$$

after the change of variables $s_{i}=2^{-u} t_{i}, i=1, \ldots, q_{0}-1$ and the definition of $\delta\left(q_{0}\right)$ in (2.1). Using the last two displays, we obtain that, as $j \rightarrow \infty$,

$$
\left\{n_{j}^{1-2 d} 2^{-2(j-u)\left(\delta\left(q_{0}\right)+K\right)} \overline{\mathbf{S}}_{n_{j}, j}\left(2^{u}+v\right)\right\}_{u, v} \stackrel{\text { fidi }}{\longrightarrow}\left\{2^{u(2 d-1)} L_{q_{0}-1}\left(\widehat{g}_{\infty}\right) f^{*}(0)^{q_{0}} Z_{d}(1)\right\}_{u, v},
$$

where $(u, v)$ take values $u=0, \ldots, p-1$ and $v=0, \ldots, 2^{u}-1$. Note that the right-hand side does not depend on $v$. By (4.2), we have $n_{j} / n_{j-u} \sim 2^{-u}$ and by (9.5), we have $\sigma_{j-u}^{2} \sim$ $q_{0} !\left(f^{*}(0)\right)^{q_{0}} L_{q_{0}}\left(\widehat{g}_{\infty}\right) 2^{2(j-u)\left(\delta\left(q_{0}\right)+K\right)}$. Thus the last display yields

$$
\left\{n_{j}^{1-2 d} \frac{1}{\sigma_{j-u}^{2}} \frac{n_{j}}{n_{j-u}} \sum_{v=0}^{2^{u}-1} \overline{\mathbf{S}}_{n_{j}, j}\left(2^{u}+v\right)\right\}_{u} \stackrel{\text { fidi }}{\longrightarrow}\left\{2^{u(2 d-1)} \frac{L_{q_{0}-1}\left(\widehat{g}_{\infty}\right)}{q_{0} ! L_{q_{0}}\left(\widehat{g}_{\infty}\right)} Z_{d}(1)\right\}_{u},
$$

where $u=0, \ldots, p-1$. Applying (9.5), we have

$$
\begin{aligned}
n_{j}^{1-2 d}\left(\frac{\widehat{\sigma}_{j-u}^{2}}{\sigma_{j-u}^{2}}-1\right) & =n_{j}^{1-2 d} \frac{1}{\sigma_{j-u}^{2}}\left(\widehat{\sigma}_{j-u}^{2}-\sigma_{j-u}^{2}\right) \\
& =n_{j}^{1-2 d} \frac{1}{\sigma_{j-u}^{2}} \frac{n_{j}}{n_{j-u}} \sum_{v=0}^{2^{u}-1} \bar{S}_{n_{j}, j}\left(2^{u}+v\right)+O_{P}\left(n_{j}^{1-2 d} / n_{j-u}\right) .
\end{aligned}
$$

By (9.4), $n_{j}^{1-2 d} / n_{j-u} \sim 2^{u} n_{j}^{-2 d} \rightarrow 0$ since $u$ is constant. Hence (4.6) follows from the last two displays.

\section{Appendix A. Technical lemmas}

A.1. Asymptotic behavior of the kernel $\widehat{\kappa}_{j}^{(p)}$. The following result provides a bound of $\widehat{\kappa}_{j}^{(p)}$ defined in (6.8), in the case where $p>0$. It is used in the proof of Proposition 7.1

Lemma A.1. Suppose that Assumptions $\boldsymbol{A}$ hold with $m=1$ and $M \geq K$, and let $0<p<$ $1 /(1-2 d)$. Then there exists some $C_{1}>0$ such that for all $\left(\xi_{1}, \xi_{2}\right) \in \mathbb{R}^{2}$ and $j \geq 0$,

$$
\left|\widehat{\kappa}_{j}^{(p)}\left(\xi_{1}, \xi_{2}\right)\right| \leq C_{1} \frac{\gamma_{j}^{2(\delta(p)+K)}}{\left(1+\gamma_{j}\left|\left\{\xi_{1}\right\}\right|\right)^{\delta(p)}\left(1+\gamma_{j}\left|\left\{\xi_{2}\right\}\right|\right)^{\delta(p)}} .
$$

Proof. By $(2 \pi)$-periodicity of $\widehat{\kappa}_{j}^{(p)}\left(\xi_{1}, \xi_{2}\right)$ along both variables $\xi_{1}$ and $\xi_{2}$, we may take $\xi_{1}, \xi_{2} \in$ $[-\pi, \pi]$. Set for all $i \in\{1, \cdots, p\}$,

$$
\mu_{i}=\gamma_{j}\left(\lambda_{i}+\cdots+\lambda_{p}\right),
$$


in the integral (6.13). Then by (1.2) and (2.16), there exists a constant $C$ independent of $j$ such that for all $\left(\xi_{1}, \xi_{2}\right) \in[-\pi, \pi]^{2}$,

$$
\left|\widehat{\kappa}_{j}^{(p)}\left(\xi_{1},-\xi_{2}\right)\right| \leq C\left\|f^{*}\right\|_{\infty}^{p} \gamma_{j}^{2 K+2 \delta(p)} \int_{-\gamma_{j} p \pi}^{\gamma_{j} p \pi} \frac{J_{p, \gamma_{j} \pi}\left(\mu_{1} ; 2 d\right) \mathrm{d} \mu_{1}}{\prod_{i=1}^{2}\left(1+\gamma_{j}\left|\left\{\mu_{1} / \gamma_{j}+\xi_{i}\right\}\right|\right)^{K+\alpha}},
$$

where $J_{p, a}$ is defined in Lemma A.6. Applying Lemma A.6 $\left(\beta=2 d, a=\gamma_{j} \pi\right)$, there exists some constant $C>0$ depending only on $p, d$ such that for any $\mu_{1} \in \mathbb{R}^{*}$,

$$
J_{p, \gamma_{j} \pi}\left(\mu_{1}, 2 d\right) \leq C\left|\mu_{1}\right|^{-(p(1-2 d)-1)}=C\left|\mu_{1}\right|^{-2 \delta(p)} .
$$

Hence there exists $C_{1}>0$ such that, for all $\left(\xi_{1}, \xi_{2}\right) \in[-\pi, \pi]^{2}$,

$$
\left|\widehat{\kappa}_{j}^{(p)}\left(\xi_{1},-\xi_{2}\right)\right| \leq C_{1} \gamma_{j}^{2 K+2 \delta(p)} \int_{-p \gamma_{j} \pi}^{p \gamma_{j} \pi} \frac{\left|\mu_{1}\right|^{-2 \delta(p)} \mathrm{d} \mu_{1}}{\prod_{i=1}^{2}\left(1+\gamma_{j}\left|\left\{\mu_{1} / \gamma_{j}+\xi_{i}\right\}\right|\right)^{K+\alpha}} .
$$

Using the Cauchy-Schwartz inequality yields

$$
\left|\widehat{\kappa}_{j}^{(p)}\left(\xi_{1},-\xi_{2}\right)\right| \leq C_{1} \gamma_{j}^{2(K+\delta(p))} \prod_{i=1}^{2}\left(\int_{-p \gamma_{j} \pi}^{p \gamma_{j} \pi} \frac{\left|\mu_{1}\right|^{-2 \delta(p)} \mathrm{d} \mu_{1}}{\left(1+\left|\gamma_{j}\left\{\mu_{1} / \gamma_{j}+\xi_{i}\right\}\right|\right)^{2(K+\alpha)}}\right)^{1 / 2} .
$$

We now use that

$$
\int_{-p \gamma_{j} \pi}^{p \gamma_{j} \pi} \frac{\left|\mu_{1}\right|^{-2 \delta(p)} \mathrm{d} \mu_{1}}{\left(1+\left|\gamma_{j}\left\{\mu_{1} / \gamma_{j}+\xi\right\}\right|\right)^{2(K+\alpha)}} \leq \sum_{|s|<(p+1) / 2} \int_{I(s)} \frac{\left|\mu_{1}\right|^{-2 \delta(p)} \mathrm{d} \mu_{1}}{\left(1+\left|\mu_{1}+\gamma_{j}(\xi-2 \pi s)\right|\right)^{2(K+\alpha)}},
$$

where $I(s)$ denotes the interval $-\gamma_{j} \xi+2 \pi s \gamma_{j}+\left[-\gamma_{j} \pi, \gamma_{j} \pi\right]$. Since we have here supposed that $\delta(p)>0$, we may apply Lemma A.5 with $d=\delta(p), q=1, a=-\gamma_{j}(\xi-2 \pi s)$ and $M_{1}=2(K+\alpha)$. We get

$$
\int_{-p \gamma_{j} \pi}^{p \gamma_{j} \pi} \frac{\left|\mu_{1}\right|^{-2 \delta(p)} \mathrm{d} \mu_{1}}{\left(1+\left|\gamma_{j}\left\{\mu_{1} / \gamma_{j}+\xi\right\}\right|\right)^{2(K+\alpha)}} \leq C \sum_{|s|<(p+1) / 2}\left(1+\gamma_{j}|\xi-2 \pi s|\right)^{-2 \delta(p)},
$$

for some positive constant $C$. Since $|\xi| \leq \pi$, we have, for any non-zero integer $s,|\xi-2 \pi s| \geq$ $(2|s|-1) \pi \geq \pi \geq|\xi|$. Hence all the terms in the last sum are at most equal to the term corresponding to $s=0$. This, with (A.3), yields (A.1).

Next we derive the limit of $\widehat{\kappa}_{j}^{(p)}$, rescaled and normalized, as $j \rightarrow \infty$. The result is used in the proof of Proposition 8.1 .

Lemma A.2. Suppose that Assumptions $\boldsymbol{A}$ hold with $m=1$ and $M \geq K$, and let $0<p<$ $1 /(1-2 d)$. Let $\left(z_{j}\right)_{j \geq 1}$ be a sequence in $\mathbb{R}^{2}$ converging to the origin. Then, as $j \rightarrow \infty$,

$$
\gamma_{j}^{p(1-2 d)-(2 K+1)} \widehat{\kappa}_{j}^{(p)}\left(z_{j} / \gamma_{j}\right) \rightarrow\left(f^{*}(0)\right)^{p} L_{p}\left(\widehat{h}_{\infty}\right),
$$

where $L_{p}\left(\widehat{h}_{\infty}\right)$ is the finite positive constant defined by 3.7).

Proof. From (2.11) and (3.7) with $M \geq K$ we get that $\left|\widehat{h}_{\infty}(\lambda)\right| /|\lambda|^{K} \leq(1+|\lambda|)^{-\alpha-K}$. The fact that $L_{p}\left(\widehat{h}_{\infty}\right)<\infty$ follows from Lemma A.5 applied with $a=0, p=q$ and $M_{1}=2(\alpha+K)$. Setting $\zeta=\gamma_{j} \lambda$ in (6.13), we get

$$
\gamma_{j}^{p(1-2 d)-(2 K+1)} \widehat{\kappa}_{j}^{(p)}(\xi)=\int_{\left(-\gamma_{j} \pi, \gamma_{j} \pi\right)^{p}} f_{j}^{(K, p)}(\zeta ; \xi) \mathrm{d}^{p} \zeta
$$


where, for all $j \geq 0, \lambda \in \mathbb{R}^{p}$ and $\xi=\left(\xi_{1}, \xi_{2}\right) \in \mathbb{R}^{2}$,

$$
f_{j}^{(K, p)}\left(\gamma_{j} \lambda ; \xi\right)=\gamma_{j}^{-2 d p-(2 K+1)} f^{\otimes p}(\lambda) \widehat{h}_{j}^{(K)}\left(\Sigma_{p}(\lambda)+\xi_{1}\right) \overline{\widehat{h}_{j}^{(K)}\left(\Sigma_{p}(\lambda)-\xi_{2}\right)} .
$$

Using (1.1), (2.10), (2.14) and $z_{j} \rightarrow 0$, we have, as $j \rightarrow \infty$,

$$
f_{j}^{(K, p)}\left(\zeta ; z_{j} / \gamma_{j}\right) \rightarrow\left(f^{*}(0)\right)^{p} \frac{\left|\widehat{h}_{\infty}\left(\zeta_{1}+\cdots+\zeta_{p}\right)\right|^{2}}{\left|\zeta_{1}+\cdots+\zeta_{p}\right|^{2 K}} \prod_{i=1}^{p}\left|\zeta_{i}\right|^{-2 d}
$$

It turns out, however, that $f_{j}^{(K, p)}\left(\zeta ; z_{j} / \gamma_{j}\right)$ cannot be uniformly bounded by an integrable function over the whole integral domain $\left(-\gamma_{j} \pi, \gamma_{j} \pi\right)^{p}$, but only on a specific subdomain, as we will show below. By (1.1) and (2.16), we have, for some constant $C>0$,

$$
\left|f_{j}^{(K, p)}\left(\zeta ; z_{j} / \gamma_{j}\right)\right| \leq C \prod_{i=1}^{p}\left|\zeta_{i}\right|^{-2 d} \sup _{|u| \leq\left|z_{j}\right|}\left(1+\left|\gamma_{j}\left\{\left(\Sigma_{p}(\zeta)+u\right) / \gamma_{j}\right\}\right|\right)^{-2(\alpha+K)} .
$$

The domains are defined using an integer $s$ by taking $\zeta$ such that $\left\{\left(\Sigma_{p}(\zeta)+u\right) / \gamma_{j}\right\}=\left(\Sigma_{p}(\zeta)+\right.$ $u) / \gamma_{j}-2 \pi s$. In fact we will use smaller domains that do not depend on $u \in\left[-\left|z_{j}\right|,\left|z_{j}\right|\right]$, namely,

$$
\Gamma_{j}^{(s)}=\left\{\zeta \in\left(-\gamma_{j} \pi, \gamma_{j} \pi\right)^{p},-\pi+2 \pi s+\left|z_{j}\right| / \gamma_{j}<\Sigma_{p}(\zeta) / \gamma_{j}<\pi+2 \pi s-\left|z_{j}\right| / \gamma_{j}\right\} .
$$

We note indeed that, for all $\zeta \in \Gamma_{j}^{(s)}$ and $u \in\left[-\left|z_{j}\right|,\left|z_{j}\right|\right],\left\{\left(\Sigma_{p}(\zeta)+u\right) / \gamma_{j}\right\}=\left(\Sigma_{p}(\zeta)+u\right) / \gamma_{j}-$ $2 \pi s$. The following set completes the partition of $\left(-\gamma_{j} \pi, \gamma_{j} \pi\right)^{p}$.

$$
\Delta_{j}=\left\{\zeta \in\left(-\gamma_{j} \pi, \gamma_{j} \pi\right)^{p}: d\left(\Sigma_{p}(\zeta) / \gamma_{j}, \pi+2 \pi \mathbb{Z}\right) \leq\left|z_{j}\right| / \gamma_{j}\right\}
$$

where $d(x, A)$ denotes the distance between a real $x$ and the set $A$. We will prove below the following facts.

(i) As $j \rightarrow \infty$, we have

$$
\int_{\Gamma_{j}^{(0)}} f_{j}^{(K, p)}\left(\zeta ; z_{j} / \gamma_{j}\right) \mathrm{d} \zeta \rightarrow\left(f^{*}(0)\right)^{p} \mathbf{L}_{p}
$$

(ii) If $|s| \geq(p+1) / 2$, for $j$ large enough, $\Gamma_{j}^{(s)}$ is an empty set.

(iii) For all $s \neq 0$, as $j \rightarrow \infty$,

$$
\int_{\Gamma_{j}^{(s)}} f_{j}^{(K, p)}\left(\zeta ; z_{j} / \gamma_{j}\right) \mathrm{d} \zeta \rightarrow 0
$$

(iv) As $j \rightarrow \infty$,

$$
\int_{\Delta_{j}} f_{j}^{(K, p)}\left(\zeta ; z_{j} / \gamma_{j}\right) \mathrm{d} \zeta \rightarrow 0
$$

To conclude the proof, we show (i), (ii), (iii) and (iv) successively.

First consider (i). It follows from (A.6), the definition of $\Gamma_{j}^{(0)}$ and $\left|z_{j}\right| \rightarrow 0$ that, for $j$ large enough,

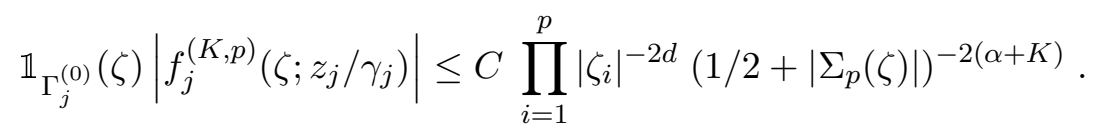

Observe that, by Lemma A.4, and since $\alpha>1 / 2, K \geq 0$ and $p(1-2 d)<1$, the right-hand side of the last display is integrable. Then (A.7) follows from (A.5) and the dominated convergence theorem.

Assertion (ii) follows from the definition of $\Gamma_{j}^{(s)}$. 
We now prove (iii) and thus take $s \neq 0$. Using (A.6) and $\left|z_{j}\right| \rightarrow 0$, we get, for all $\zeta \in \Gamma_{j}^{(s)}$ and $j$ large enough,

$$
\left|f_{j}^{(K, p)}\left(\zeta ; z_{j} / \gamma_{j}\right)\right| \leq C \prod_{i=1}^{p}\left|\zeta_{i}\right|^{-2 d}\left(1 / 2+\left|\Sigma_{p}(\zeta)-2 \pi s \gamma_{j}\right|\right)^{-2(\alpha+K)} .
$$

The limit (A.8) then follows from Lemma A.5 applied with $q=p, M_{1}=2(K+\alpha)$ and $a=2 \pi \gamma_{j} s$.

Finally we prove Assertion (iv). In this case, we observe that (A.6) and implies

$$
\left|f_{j}^{(K, p)}\left(\zeta ; z_{j} / \gamma_{j}\right)\right| \leq C \prod_{i=1}^{p}\left|\zeta_{i}\right|^{-2 d} .
$$

This bound and Lemma A.4 yields

$$
\int_{\Delta_{j}} f_{j}^{(K, p)}\left(\zeta ; z_{j} / \gamma_{j}\right) \mathrm{d} \zeta \leq C \int_{-p \gamma_{j} \pi}^{p \gamma_{j} \pi} \mathbb{1}_{d\left(t / \gamma_{j}, \pi+2 \pi \mathbb{Z}\right) \leq\left|z_{j}\right| / \gamma_{j}} \mathrm{~d} t=O\left(\left|z_{j}\right|\right) .
$$

Hence, since $\left|z_{j}\right| \rightarrow 0$, we obtain (A.9) and the proof is achieved.

\section{A.2. Other technical lemmas.}

Lemma A.3. Define the Dirichlet kernel $D_{n}$ as in (6.7). Then

$$
\sup _{\theta \in \mathbb{R}} \sup _{n \geq 1}(1+|n\{\theta / n\}|)\left|D_{n}(\theta / n)\right|<\infty .
$$

Proof. We observe that $\left|\mathrm{e}^{\mathrm{i} \lambda}-1\right| \geq 2|\{\lambda\}| / \pi$. Hence, for all $\theta \in \mathbb{R}$,

$$
\left|D_{n}(\theta / n)\right| \leq \frac{\pi}{2} \frac{\left|\mathrm{e}^{\mathrm{i} \theta}-1\right|}{|n\{\theta / n\}|}=\frac{\pi}{2} \frac{\left|\mathrm{e}^{\mathrm{i} n\{\theta / n\}}-1\right|}{|n\{\theta / n\}|} .
$$

(We use the usual continuous extension convention $\left.\left(\mathrm{e}^{\mathrm{i} 0}-1\right) / 0=1\right)$. Now, using that $\left|e^{\mathrm{i} u}-1\right| \leq$ $2|u| /(1+|u|)$ on $u \in \mathbb{R}$, we get (A.10).

Lemma A.4. Let $p$ be a positive integer and $f: \mathbb{R} \rightarrow \mathbb{R}_{+}$. Then, for any $\beta \in \mathbb{R}^{q}$,

$$
\int_{\mathbb{R}^{q}} f\left(y_{1}+\cdots+y_{q}\right) \prod_{i=1}^{q}\left|y_{i}\right|^{\beta_{i}} \mathrm{~d} y_{1} \cdots \mathrm{d} y_{q}=\Gamma \times \int_{\mathbb{R}} f(s)|s|^{q-1+\beta_{1}+\cdots+\beta_{q}} \mathrm{~d} s,
$$

where, for all $i \in\{1, \cdots, q\}, B_{i}=\beta_{i}+\cdots+\beta_{q}$ and

$$
\Gamma=\prod_{i=2}^{q}\left(\int_{\mathbb{R}}|t|^{q-i+B_{i}}|1-t|^{\beta_{i-1}} \mathrm{~d} t\right) .
$$

(We note that $\Gamma$ may be infinite in which case (A.11) holds with the convention $\infty \times 0=0$ ).

Proof. This follows from Lemma 8.3 in [11].

Lemma A.5. Let $d \in(0,1 / 2)$ and $q$ be a positive integer such that $q<1 /(1-2 d)$. Let $M_{1}>1$. Set for any $a \in \mathbb{R}$,

$$
J_{q}\left(a ; M_{1} ; d\right)=\int_{\mathbb{R}^{q}}\left(1+\left|\Sigma_{q}(\zeta)-a\right|\right)^{-M_{1}} \prod_{i=1}^{q}\left|\zeta_{i}\right|^{-2 d} \mathrm{~d} \zeta .
$$


Then one has

$$
\sup _{a \in \mathbb{R}}(1+|a|)^{1-q(1-2 d)} J_{q}\left(a ; M_{1} ; d\right)<\infty
$$

In particular,

$$
J_{q}\left(0 ; M_{1} ; d\right)<\infty
$$

and

$$
J_{q}\left(a ; M_{1} ; d\right)=O\left(|a|^{-(1-q(1-2 d)}\right) \quad \text { as } a \rightarrow \infty .
$$

Proof. This follows from Lemma 8.4 of in [11].

Lemma A.6. Define, for all $a>0$ and $\beta_{1} \in(0,1)$,

$$
J_{1, a}\left(s_{1} ; \beta_{1}\right)=\left|s_{1}\right|^{-\beta_{1}}, \quad s_{1} \in \mathbb{R},
$$

and, for any integer $m \geq 2$ and $\beta=\left(\beta_{1}, \cdots, \beta_{m}\right) \in(0,1)^{m}$,

$$
J_{m, a}\left(s_{1} ; \beta\right)=\int_{s_{2}=-(m-1) a}^{(m-1) a} \ldots \int_{s_{m}=-a}^{a} \prod_{i=2}^{m}\left|s_{i-1}-s_{i}\right|^{-\beta_{i-1}}\left|s_{m}\right|^{-\beta_{m}} \mathrm{~d} s_{m} \ldots \mathrm{d} s_{2}, \quad s_{1} \in \mathbb{R} .
$$

Then

(i) if $\beta_{1}+\cdots+\beta_{m}>m-1$, one has

$$
C_{m}(\beta)=\sup _{a>0} \sup _{s_{1} \in \mathbb{R}}\left(\left|s_{1}\right|^{-\left(m-1-\left(\beta_{1}+\cdots+\beta_{m}\right)\right)} J_{m, a}\left(s_{1} ; \beta\right)\right)<\infty,
$$

(ii) if $\beta_{1}+\cdots+\beta_{m}=m-1$, one has

$$
C_{m}(\beta)=\sup _{a>0} \sup _{\left|s_{1}\right| \leq m a}\left(\frac{1}{1+\log \left(m a /\left|s_{1}\right|\right)} J_{m, a}\left(s_{1} ; \beta\right)\right)<\infty,
$$

(iii) if there exists $q \in\{2, \ldots, m\}$ such that $\beta_{q}+\cdots+\beta_{m}=m-q$, one has

$$
C_{m}(\beta)=\sup _{a>0} \sup _{\left|s_{1}\right| \leq m a}\left(\frac{a^{-\left(q-1-\left(\beta_{1}+\cdots+\beta_{q-1}\right)\right)}}{1+\log \left(m a /\left|s_{1}\right|\right)} J_{m, a}\left(s_{1} ; \beta\right)\right)<\infty,
$$

(iv) if $\beta_{1}+\cdots+\beta_{m}<m-1$ and for all $q \in\{1, \ldots, m-1\}$, we have $\beta_{q}+\cdots+\beta_{m} \neq m-q$, one has

$$
C_{m}(\beta)=\sup _{a>0} \sup _{\left|s_{1}\right| \leq m a}\left(a^{-\left(m-1-\left(\beta_{1}+\cdots+\beta_{m}\right)\right)} J_{m, a}\left(s_{1} ; \beta\right)\right)<\infty .
$$

Remark A.1. We observe that Cases (ii),(iii) and (iv) can be put together as the following formula, valid for all $\beta \in(0,1)^{m}$ such that $\beta_{1}+\cdots+\beta_{m} \leq m-1$,

$$
C_{m}(\beta)=\sup _{a>0} \sup _{\left|s_{1}\right| \leq m a}\left(\frac{a^{-\left(q-1-\left(\beta_{1}+\cdots+\beta_{q-1}\right)\right)}}{\left\{1+\log \left(m a /\left|s_{1}\right|\right)\right\}^{\varepsilon}} J_{m, a}\left(s_{1} ; \beta\right)\right)<\infty,
$$

where $\varepsilon=1$ if there exists $q \in\{1, \ldots, m\}$ such that $\beta_{q}+\cdots+\beta_{m}=m-q$, and $\varepsilon=0$ otherwise. We may also include case (i) as follows,

$$
C_{m}(\beta)=\sup _{a>0} \sup _{\left|s_{1}\right| \leq m a}\left(\frac{a^{-\left(m-1-\left(\beta_{1}+\cdots+\beta_{m}\right)\right)_{+}}\left|s_{1}\right|^{\left(m-1-\left(\beta_{1}+\cdots+\beta_{m}\right)\right)_{-}}}{\left\{1+\log \left(m a /\left|s_{1}\right|\right)\right\}^{\varepsilon}} J_{m, a}\left(s_{1} ; \beta\right)\right)<\infty,
$$

where $\varepsilon$ is as above, and $a_{+}=\max (a, 0)$ and $a_{-}=\max (-a, 0)$ denote the positive and negative parts of a, respectively. 
Proof. Observe first that for all $m \geq 1$,

$$
J_{m, a}\left(s_{1} ; \beta\right)=\int_{s_{2}=-(m-1) a}^{(m-1) a}\left|s_{2}-s_{1}\right|^{-\beta_{1}} J_{m-1, a}\left(s_{2} ; \beta^{\prime}\right) \mathrm{d} s_{2},
$$

where $\beta^{\prime}=\left(\beta_{2}, \ldots, \beta_{m}\right)$. The bounds $C_{m}(\beta)$ in the different cases will follow by induction on $m$.

Let us first prove the result for $m=1$ and $m=2$. If $m=1, \beta=\beta_{1} \in(0,1)$ only satisfies the condition of Case (i) and, since $J_{1, a}$ is given by (A.13), the result holds for $m=1$. Assume now that $m=2$ and $s_{1} \neq 0$ and set $s_{2}=v\left|s_{1}\right|$. Then

$$
J_{2, a}\left(s_{1} ; \beta\right)=\left|s_{1}\right|^{1-\left(\beta_{1}+\beta_{2}\right)} \int_{-a /\left|s_{1}\right|}^{a /\left|s_{1}\right|} \frac{\mathrm{d} v}{|1-v|^{\beta_{1}}|v|^{\beta_{2}}} .
$$

In the case $\beta_{1}+\beta_{2}>1$, we are in Case (i). Since $\int_{\mathbb{R}} \frac{\mathrm{d} v}{|1-v|^{\beta_{1}|v|^{\beta_{2}}}}$ is finite, the required upper bound holds. If $\beta_{1}+\beta_{2} \leq 1$, we are either in Case (ii) or (iv) and the result follows from the following bounds valid for some constant $c$ depending only on $\beta$, if $\beta_{1}+\beta_{2}<1$ and $x \geq 1 / 2$,

$$
\int_{-x}^{x} \frac{\mathrm{d} v}{|1-v|^{\beta_{1}}|v|^{\beta_{2}}} \leq c x^{1-\left(\beta_{1}+\beta_{2}\right)}
$$

and, if $\beta_{1}+\beta_{2}=1$ and $x \geq 1 / 2$,

$$
\int_{-x}^{x} \frac{\mathrm{d} v}{|1-v|^{\beta_{1}}|v|^{\beta_{2}}} \leq C(1+\log (2 x)) .
$$

This prove the result for $m=2$ because $x=a /\left|s_{1}\right| \geq 1 / 2$.

Let us now assume that the result holds for some positive integer $m-1$ and prove it for $m$. We consider two different cases.

(1) If $\beta$ satisfies the conditions of Case (i), Case (ii), or Case (iv) then $\beta^{\prime}$ satisfies the conditions of Case (i) or (iv). Then by (A.17) and the induction assumption,

$$
J_{m, a}\left(s_{1} ; \beta\right) \leq C_{m-1}\left(\beta^{\prime}\right) a^{\left[m-2-\Sigma_{m-1}\left(\beta^{\prime}\right)\right]_{+}} \int_{-(m-1) a}^{(m-1) a}\left|s_{2}-s_{1}\right|^{-\beta_{1}}\left|s_{2}\right|^{\left.-\left[\Sigma_{m-1}\left(\beta^{\prime}\right)-(m-2)\right)\right]_{+}} \mathrm{d} s_{2},
$$

where $\Sigma_{m-1}\left(\beta^{\prime}\right)=\beta_{2}+\cdots+\beta_{m}$ and $[x]_{+}=\max (x, 0)$. If $\Sigma_{m-1}\left(\beta^{\prime}\right)<m-2$ (so that $\beta$ satisfies (iv)), the conclusion follows from the following bound valid for some constant $c$ depending only on $\beta$ and all $x \geq\left|s_{1}\right| / 2$,

$$
\int_{-x}^{x}\left|s_{2}-s_{1}\right|^{-\beta_{1}} \mathrm{~d} s_{2}=\left|s_{1}\right|^{1-\beta_{1}} \int_{-x /\left|s_{1}\right|}^{x /\left|s_{1}\right|}|u-1|^{-\beta_{1}} \mathrm{~d} u \leq c x^{1-\beta_{1}} .
$$

Now if $\Sigma_{m-1}\left(\beta^{\prime}\right)>m-2$, we observe that

$$
\int_{-(m-1) a}^{(m-1) a}\left|s_{2}-s_{1}\right|^{-\beta_{1}}\left|s_{2}\right|^{-\left[\beta_{2}+\cdots+\beta_{m}-(m-2)\right]} \mathrm{d} s_{2}=J_{2,(m-1) a}\left(s_{1} ; \beta_{1}, \beta_{2}+\cdots+\beta_{m}-(m-2)\right) \text {. }
$$

The upper bound of $J_{m, a}\left(s_{1} ; \beta\right)$ then follows from the case $m=2$.

(2) If $\beta$ satisfies the condition of Case (iii), then $\beta^{\prime}$ either satisfies the conditions of Case (ii) or (iii). The proof is exactly similar to this just above up to a logarithmic correction. 
Lemma A.7. Let $S>1$ and $\left(\beta_{1}, \beta_{2}\right) \in[0,1)^{2}$ such that $\beta_{1}+\beta_{2}<1$, and set $g_{i}(t)=$ $|t|^{-\beta_{i}}(1+|t|)^{\beta_{i}-S}$. Then

$$
\sup _{\nu \geq 0}\left(\nu \int_{\mathbb{R}^{2}}\left(1+\nu\left|\left\{w_{1}+w_{2}\right\}\right|\right)^{-2} g_{1}\left(w_{1}\right) g_{2}\left(w_{2}\right) \mathrm{d} w\right)<\infty .
$$

Proof. Denote by $J(\nu)$ the quantity in parentheses in (A.19). We denote here by $C$ a positive constant that may change from line to line, but whose value does not depend on $\nu$. Setting $u=w_{1}+w_{2}$ in the integral with respect to $w_{1}$ and then integrating with respect to $w_{2}$, Lemma 8.1 in [11] yields

$$
J(\nu) \leq C \nu \int_{u \in \mathbb{R}}(1+\nu|\{u\}|)^{-2}(1+|u|)^{-S} \mathrm{~d} u .
$$

Since the integral is bounded independently of $\nu, J$ is bounded on compact subsets of $[0, \infty)$, hence we may consider $\nu \geq 2$ in the remainder of the proof. We shall use the bound $1+x \geq$ $\max (1, x)$ for $x \geq 0$. Splitting the integral of the last display on the two domains defined by the position of $|\{u\}|$ with respect to $\nu^{-1}$, we get $J(\nu) \leq C\left(J_{1}(\nu)+J_{2}(\nu)\right)$, with

$$
J_{1}(\nu)=\nu \int_{|\{u\}| \leq \nu^{-1}}(1+|u|)^{-S} \mathrm{~d} u,
$$

and

$$
J_{2}(\nu)=\nu^{-1} \int_{|\{u\}| \geq \nu^{-1}}|\{u\}|^{-2}(1+|u|)^{-S} \mathrm{~d} u .
$$

We have

$$
J_{1}(\nu)=\nu \sum_{k \in \mathbb{Z}} \int_{2 k \pi-\nu^{-1}}^{2 k \pi+\nu^{-1}}(1+|u|)^{-S} \mathrm{~d} u .
$$

For $\nu \geq 2$ the integral in the parentheses of the last display is less than $2 \nu^{-1}(1 / 2+|2 k \pi|)^{-S}$. Since $S>1$, we get that $J_{1}(u)$ is bounded over the domain $\nu \geq 2$.

It remains to prove that $J_{2}(\nu)$ is bounded for $\nu$ large enough. We have, setting $v=u-2 k \pi$ for each $k$,

$$
J_{2}(\nu)=\nu^{-1} \sum_{k \in \mathbb{Z}} \int_{\nu^{-1} \leq|v| \leq \pi}|v|^{-2}(1+|2 k \pi+v|)^{-S} \mathrm{~d} v
$$

Now since

$$
\sup _{v \in \mathbb{R}} \sum_{k \in \mathbb{Z}}(1 / 2+|2 k \pi+v|)^{-S}<\infty
$$

we get by inverting the integral with the summation,

$$
J_{2}(\nu) \leq C \nu^{-1} \int_{\nu^{-1} \leq|v| \leq \pi}|v|^{-2} \mathrm{~d} v .
$$

Hence $J_{2}$ is bounded over the domain $\nu \geq 2$, completing the proof. 


\section{ApPendix B. IntEgRAL REPRESENTATIONS}

It is convenient to use an integral representation in the spectral domain to represent the random processes (see for example [23, 27]). The stationary Gaussian process $\left\{X_{k}, k \in \mathbb{Z}\right\}$ with spectral density (1.2) can be written as

$$
X_{\ell}=\int_{-\pi}^{\pi} \mathrm{e}^{\mathrm{i} \lambda \ell} f^{1 / 2}(\lambda) \mathrm{d} \widehat{W}(\lambda)=\int_{-\pi}^{\pi} \frac{\mathrm{e}^{\mathrm{i} \lambda \ell} f^{* 1 / 2}(\lambda)}{\left|1-\mathrm{e}^{-\mathrm{i} \lambda}\right|^{d}} \mathrm{~d} \widehat{W}(\lambda), \quad \ell \in \mathbb{Z} .
$$

This is a special case of

$$
\widehat{I}(g)=\int_{\mathbb{R}} g(x) \mathrm{d} \widehat{W}(x),
$$

where $\widehat{W}(\cdot)$ is a complex-valued Gaussian random measure satisfying, for any Borel sets $A$ and $B$ in $\mathbb{R}, \mathbb{E}(\widehat{W}(A))=0, \mathbb{E}(\widehat{W}(A) \widehat{\widehat{W}(B)})=|A \cap B|$ and $\widehat{W}(A)=\widehat{\widehat{W}(-A)}$. The integral (B.2) is defined for any function $g \in L^{2}(\mathbb{R})$ and one has the isometry

$$
\mathbb{E}\left(|\widehat{I}(g)|^{2}\right)=\int_{\mathbb{R}}|g(x)|^{2} \mathrm{~d} x .
$$

The integral $\widehat{I}(g)$, moreover, is real-valued if $g(x)=\overline{g(-x)}$.

We shall also consider multiple Itô-Wiener integrals

$$
\widehat{I}_{q}(g)=\int_{\mathbb{R}^{q}}^{\prime \prime} g\left(\lambda_{1}, \cdots, \lambda_{q}\right) \mathrm{d} \widehat{W}\left(\lambda_{1}\right) \cdots \mathrm{d} \widehat{W}\left(\lambda_{q}\right)
$$

where the double prime indicates that one does not integrate on hyperdiagonals $\lambda_{i}= \pm \lambda_{j}, i \neq$ $j$. The integrals $\widehat{I}_{q}(g)$ are handy because we will be able to expand our non-linear functions $G\left(X_{k}\right)$ introduced in Section 1 in multiple integrals of this type.

These multiples integrals are defined for $g \in \overline{L^{2}}\left(\mathbb{R}^{q}, \mathbb{C}\right)$, the space of complex valued functions defined on $\mathbb{R}^{q}$ satisfying

$$
\begin{gathered}
g\left(-x_{1}, \cdots,-x_{q}\right)=\overline{g\left(x_{1}, \cdots, x_{q}\right)} \text { for }\left(x_{1}, \cdots, x_{q}\right) \in \mathbb{R}^{q}, \\
\|g\|_{L^{2}}^{2}:=\int_{\mathbb{R}^{q}}\left|g\left(x_{1}, \cdots, x_{q}\right)\right|^{2} \mathrm{~d} x_{1} \cdots \mathrm{d} x_{q}<\infty .
\end{gathered}
$$

The integral $\widehat{I}_{q}(g)$ is real valued and verifies $\widehat{I}_{q}(g)=\widehat{I}_{q}(\tilde{g})$, where

$$
\tilde{g}\left(x_{1}, \cdots, x_{q}\right)=\frac{1}{q !} \sum_{\sigma} g\left(x_{\sigma(1)}, \cdots, x_{\sigma(q)}\right) .
$$

Here the sum is over all permutations of $\{1, \ldots, q\}$.

$$
\mathbb{E}\left(\widehat{I}_{q}\left(g_{1}\right) \widehat{I}_{q^{\prime}}\left(g_{2}\right)\right)= \begin{cases}q !\left\langle\tilde{g_{1}}, \tilde{g_{2}}\right\rangle_{L^{2}} & \text { if } q=q^{\prime} \\ 0 & \text { if } q \neq q^{\prime}\end{cases}
$$

Hermite polynomials are related to multiple integrals as follows : if $X=\int_{\mathbb{R}} g(x) \mathrm{d} \widehat{W}(x)$ with $\mathbb{E}\left(X^{2}\right)=\int_{\mathbb{R}}|g(x)|^{2} \mathrm{~d} x=1$ and $g(x)=\overline{g(-x)}$ so that $X$ has unit variance and is real-valued, then

$$
H_{q}(X)=\widehat{I}_{q}\left(g^{\otimes q}\right)=\int_{\mathbb{R}^{q}}^{\prime \prime} g\left(x_{1}\right) \cdots g\left(x_{q}\right) \mathrm{d} \widehat{W}\left(x_{1}\right) \cdots \mathrm{d} \widehat{W}\left(x_{q}\right)
$$


Since $X$ has unit variance, one has for any $\ell \in \mathbb{Z}$,

$$
\begin{aligned}
H_{q}\left(X_{\ell}\right) & =H_{q}\left(\int_{-\pi}^{\pi} \mathrm{e}^{\mathrm{i} \xi \ell} f^{1 / 2}(\xi) \mathrm{d} \widehat{W}(\xi)\right) \\
& =\int_{(-\pi, \pi]^{q}}^{\prime \prime} \mathrm{e}^{\mathrm{i} \ell\left(\xi_{1}+\cdots+\xi_{q}\right)} \times\left(f^{1 / 2}\left(\xi_{1}\right) \times \cdots \times f^{1 / 2}\left(\xi_{q}\right)\right) \mathrm{d} \widehat{W}\left(\xi_{1}\right) \cdots \mathrm{d} \widehat{W}\left(\xi_{q}\right) .
\end{aligned}
$$

Then by (2.13), we have

$$
W_{j, k}=\sum_{\ell \in \mathbb{Z}} h_{j}^{(K)}\left(\gamma_{j} k-\ell\right) H_{q_{0}}\left(X_{\ell}\right)=\widehat{I}_{q_{0}}\left(f_{j, k}^{\left(q_{0}\right)}\right)
$$

with

$$
f_{j, k}^{(q)}\left(\xi_{1}, \cdots, \xi_{q}\right)=\mathrm{e}^{\mathrm{i} k \gamma_{j}\left(\xi_{1}+\cdots+\xi_{q}\right)} \times \widehat{h}_{j}^{(K)}\left(\xi_{1}+\cdots+\xi_{q}\right) f^{1 / 2}\left(\xi_{1}\right) \cdots f^{1 / 2}\left(\xi_{q}\right) \mathbb{1}_{(-\pi, \pi)}^{\otimes q}(\xi),
$$

because by (2.5),

$$
\begin{aligned}
\sum_{\ell \in \mathbb{Z}} \mathrm{e}^{\mathrm{i} \ell\left(\xi_{1}+\cdots+\xi_{q}\right)} h_{j}^{(K)}\left(\gamma_{j} k-\ell\right) & =\mathrm{e}^{\mathrm{i} \gamma_{j} k\left(\xi_{1}+\cdots+\xi_{q}\right)} \sum_{u \in \mathbb{Z}} \mathrm{e}^{-\mathrm{i} u\left(\xi_{1}+\cdots+\xi_{q}\right)} h_{j}^{(K)}(u) \\
& =\mathrm{e}^{\mathrm{i} \gamma_{j} k\left(\xi_{1}+\cdots+\xi_{q}\right)} \widehat{h}_{j}^{(K)}\left(\xi_{1}+\cdots+\xi_{q}\right) .
\end{aligned}
$$

Observe now that since we have defined the Fourier transform of a function $f \in L^{2}\left(\mathbb{R}^{q}\right)$ as

$$
\widehat{f}(\xi)=\int_{\mathbb{R}^{q}} f(x) \mathrm{e}^{-\mathrm{i} x \xi} \mathrm{d} x \in \overline{L^{2}}\left(\mathbb{R}^{q}\right),
$$

we have by Parseval

$$
\|\widehat{f}\|_{L^{2}\left(\mathbb{R}^{q}\right)}^{2}=(2 \pi)^{q}\|f\|_{L^{2}\left(\mathbb{R}^{q}\right)}^{2} .
$$

Since moreover, $\mathbb{E}\left(I_{q}(\widehat{f})^{2}\right)=\|\widehat{f}\|_{L^{2}\left(\mathbb{R}^{q}\right)}$ and $\mathbb{E}\left(\widehat{I}_{q}(f)^{2}\right)=\|f\|_{L^{2}\left(\mathbb{R}^{q}\right)}^{2}$, we have

$$
I_{q}(\widehat{f}) \stackrel{(\mathcal{L})}{=}(2 \pi)^{q / 2} \widehat{I}_{q}(f) .
$$

The following proposition is an extension to our complex-valued setting of a corresponding result in [27] for multiple integrals in a real-valued setting. Since it plays an essential role, we provide a proof for the convenience of the reader.

Proposition B.1. Let $\left(q, q^{\prime}\right) \in \mathbb{N}^{2}$. Assume that $f, g$ are two symmetric functions belonging respectively to $\overline{L^{2}}\left(\mathbb{R}^{q}\right)$ and $\overline{L^{2}}\left(\mathbb{R}^{q^{\prime}}\right)$ then the following product formula holds :

$$
\widehat{I}_{q}(f) \widehat{I}_{q^{\prime}}(g)=\sum_{p=0}^{q \wedge q^{\prime}} p !\left(\begin{array}{l}
q \\
p
\end{array}\right)\left(\begin{array}{l}
q^{\prime} \\
p
\end{array}\right) \widehat{I}_{q+q^{\prime}-2 p}\left(f \bar{\otimes}_{p} g\right),
$$

where $f \bar{\otimes}_{0} g=f \otimes g$ is the usual tensor product and, for any $p \in\left\{1, \cdots, q \wedge q^{\prime}\right\}$,

$$
\left(f \bar{\otimes}_{p} g\right)\left(t_{1}, \cdots, t_{q+q^{\prime}-2 p}\right)=\int_{\mathbb{R}^{p}} f\left(t_{1}, \cdots, t_{q-p}, s\right) g\left(t_{q-p+1}, \cdots, t_{q+q^{\prime}-2 p},-s\right) \mathrm{d}^{p} s .
$$

Proof. We first assume that $f$ and $g$ are of the form

$$
f=f_{1} \otimes f_{2}, g=g_{1} \otimes g_{2},
$$


where $f_{1}, f_{2}, g_{1}, g_{2}$ belong respectively to $\overline{L^{2}}\left(\mathbb{R}^{q-p}, \mathbb{C}\right), \overline{L^{2}}\left(\mathbb{R}^{q^{\prime}-p}, \mathbb{C}\right), \overline{L^{2}}\left(\mathbb{R}^{p}, \mathbb{C}\right), \overline{L^{2}}\left(\mathbb{R}^{p}, \mathbb{C}\right)$. In that special case, using that for any $q \geq 1$ and any $f \in \overline{L^{2}}\left(\mathbb{R}^{q}\right), \widehat{I}_{q}(f)=(2 \pi)^{-q / 2} I_{q}(\widehat{f})$ by (B.9), one has

$$
\widehat{I}_{q}(f) \widehat{I}_{q^{\prime}}(g)=\widehat{I}_{q}\left(f_{1} \otimes f_{2}\right) \widehat{I}_{q^{\prime}}\left(g_{1} \otimes g_{2}\right)=(2 \pi)^{-\left(q+q^{\prime}\right) / 2} I_{q}\left(\widehat{f_{1}} \otimes \widehat{f}_{2}\right) I_{q^{\prime}}\left(\widehat{g_{1}} \otimes \widehat{g_{2}}\right) .
$$

The assumptions on functions $f_{1}, f_{2}, g_{1}, g_{2}$ imply that their Fourier transform $\widehat{f_{1}}, \widehat{f_{2}}, \widehat{g_{1}}, \widehat{g_{2}}$ are real-valued functions belonging respectively to $L^{2}\left(\mathbb{R}^{q-p}, \mathbb{R}\right), L^{2}\left(\mathbb{R}^{q^{\prime}-p}, \mathbb{R}\right), L^{2}\left(\mathbb{R}^{p}, \mathbb{R}\right)$ and $L^{2}\left(\mathbb{R}^{\ell}, \mathbb{R}\right)$. Then one can apply the usual product formula for multiple Wiener-Itô integrals (see for example [27]) and deduce that :

$$
I_{q}\left(\widehat{f_{1}} \otimes \widehat{f_{2}}\right) I_{q^{\prime}}\left(\widehat{g_{1}} \otimes \widehat{g_{2}}\right)=\sum_{p=0}^{q \wedge q^{\prime}} p !\left(\begin{array}{l}
q \\
p
\end{array}\right)\left(\begin{array}{l}
q^{\prime} \\
p
\end{array}\right) I_{q+q^{\prime}-2 p}\left(\left(\widehat{f_{1}} \otimes \widehat{f_{2}}\right) \otimes_{p}\left(\widehat{g_{1}} \otimes \widehat{g_{2}}\right)\right) .
$$

Note now that for any $p$

$$
\begin{aligned}
\left(\widehat{f_{1}} \otimes \widehat{f_{2}}\right) \otimes_{p}\left(\widehat{g_{1}} \otimes \widehat{g_{2}}\right) & =\int_{\mathbb{R}^{p}} \widehat{f_{1}}\left(t_{1}, \cdots, t_{q-p}\right) \widehat{f_{2}}(s) \widehat{g_{1}}\left(t_{q-p+1}, \cdots, t_{q+q^{\prime}-2 p}\right) \widehat{g_{2}}(s) \mathrm{d} s \\
& =\widehat{f}_{1}\left(t_{1}, \cdots, t_{q-p}\right) \widehat{g_{1}}\left(t_{q-p+1}, \cdots, t_{q+q^{\prime}-2 p}\right) \int_{\mathbb{R}^{p}} \widehat{f_{2}}(s) \widehat{g_{2}}(s) \mathrm{d} s \\
& =\widehat{f}_{1}\left(t_{1}, \cdots, t_{q-p}\right) \widehat{g_{1}}\left(t_{q-p+1}, \cdots, t_{q+q^{\prime}-2 p}\right)(2 \pi)^{p} \int_{\mathbb{R}^{p}} f_{2}(t) \widehat{g_{2}(t)} \mathrm{d} t \\
& =\widehat{f}_{1}\left(t_{1}, \cdots, t_{q-p}\right) \widehat{g_{1}}\left(t_{q-p+1}, \cdots, t_{q+q^{\prime}-2 p}\right)(2 \pi)^{p} \int_{\mathbb{R}^{p}} f_{2}(t) g_{2}(-t) \mathrm{d} t,
\end{aligned}
$$

since $\overline{g_{2}(t)}=g_{2}(-t)$ and using the Parseval's formula. Hence

$$
\begin{aligned}
I_{q+q^{\prime}-2 p}\left(\left(\widehat{f_{1}} \otimes \widehat{f}_{2}\right) \otimes_{p}\left(\widehat{g_{1}} \otimes \widehat{g}_{2}\right)\right) & =(2 \pi)^{p}\left(\int_{\mathbb{R}^{p}} f_{2}(t) g_{2}(-t) d t\right) \times I_{q+q^{\prime}-2 p}\left(\widehat{f_{1}} \otimes \widehat{g}_{1}\right) \\
& =(2 \pi)^{p}\left(\int_{\mathbb{R}^{p}} f_{2}(t) g_{2}(-t) d t\right) \times I_{q+q^{\prime}-2 p}\left(\widehat{f_{1} \otimes g_{1}}\right) \\
& =(2 \pi)^{p}\left(\int_{\mathbb{R}^{p}} f_{2}(t) g_{2}(-t) d t\right) \times(2 \pi)^{\left(q+q^{\prime}-2 p\right) / 2} \widehat{I}_{q+q^{\prime}-2 p}\left(f_{1} \otimes g_{1}\right) \\
& =(2 \pi)^{\left(q+q^{\prime}\right) / 2}\left(\int_{\mathbb{R}^{p}} f_{2}(t) g_{2}(-t) d t\right) \widehat{I}_{q+q^{\prime}-2 p}\left(f_{1} \otimes g_{1}\right) \\
& =(2 \pi)^{\left(q+q^{\prime}\right) / 2} \widehat{I}_{q+q^{\prime}-2 p}\left(f \bar{\otimes}_{p} g\right) .
\end{aligned}
$$

Using the last equality and equations $(\mathrm{B} .12),(\mathrm{B} .13)$, we get the claimed results for this special case. The conclusion for general $f$ and $g$ follows using the density of $L^{2}\left(\mathbb{R}^{q-p}, \mathbb{R}\right) \otimes L^{2}\left(\mathbb{R}^{p}, \mathbb{R}\right)$ in $L^{2}\left(\mathbb{R}^{q}, \mathbb{R}\right)$.

\section{REFERENCES}

[1] P. Abry and V. Pipiras. Wavelet-based synthesis of the Rosenblatt process. Eurasip Signal Processing, 86(19):2326-2339, 2006.

[2] P. Abry and D. Veitch. Wavelet analysis of long-range-dependent traffic. IEEE Trans. Inform. Theory, 44(1):2-15, 1998. ISSN 0018-9448.

[3] P. Abry, D. Veitch, and P. Flandrin. Long-range dependence: revisiting aggregation with wavelets. J. Time Ser. Anal., 19(3):253-266, 1998. ISSN 0143-9782. 
[4] P. Abry, Helgason H., and V. Pipiras. Wavelet-based analysis of non-Gaussian long-range dependent processes and estimation of the Hurst parameter. Lithuanian Mathematical Journal, 2011. To appear.

[5] J.-M. Bardet. Statistical study of the wavelet analysis of fractional Brownian motion. IEEE Trans. Inform. Theory, 48(4):991-999, 2002. ISSN 0018-9448.

[6] J.-M. Bardet and C. A. Tudor. A wavelet analysis of the Rosenblatt process: chaos expansion and estimation of the self-similarity parameter. Stochastic Process. Appl., 120(12):2331-2362, 2010. ISSN 0304-4149. doi: 10.1016/j.spa.2010.08.003. URL http://dx.doi.org/10.1016/j.spa.2010.08.003.

[7] J.-M. Bardet, G. Lang, E. Moulines, and P. Soulier. Wavelet estimator of long-range dependent processes. Stat. Inference Stoch. Process., 3(1-2):85-99, 2000. ISSN 13870874. 19th "Rencontres Franco-Belges de Statisticiens" (Marseille, 1998).

[8] J.M. Bardet, H. Bibi, and A. Jouini. Adaptive wavelet based estimator of the memory parameter for stationary gaussian processes. Bernoulli, 14:691-724, 2008.

[9] J.-C. Breton and I. Nourdin. Error bounds on the non-normal approximation of hermite power variations of fractional brownian motion. Electronic Communications in Probability, 13:482-493, 2008.

[10] A. Chronopoulou, C. Tudor, and F. Viens. Self-similarity parameter estimation and reproduction property for non-gaussian Hermite processes. Communications on Stochastic Analysis, 5:161-185, 2011.

[11] M. Clausel, F. Roueff, M. S. Taqqu, and C. Tudor. Large scale behavior of wavelet coefficients of non-linear subordinated processes with long memory. Applied and Computational Harmonic Analysis, 2011. ISSN 1063-5203. doi: 10.1016/j.acha.2011.04.003. URL http://www.sciencedirect.com/science/article/pii/S1063520311000601.

[12] M. Clausel, F. Roueff, M. S. Taqqu, and C. Tudor. High order chaotic limits of wavelet scalograms under long-range dependence. Technical report, Hal-Institut Telecom, 2012. URL http://hal-institut-telecom . archives-ouvertes.fr/hal-00662317.

[13] R. L. Dobrushin and P. Major. Non-central limit theorems for nonlinear functionals of Gaussian fields. Z. Wahrsch. Verw. Gebiete, 50(1):27-52, 1979. ISSN 0044-3719. doi: 10.1007/BF00535673. URL http://dx.doi.org/10.1007/BF00535673.

[14] P. Embrechts and M. Maejima. Selfsimilar processes. Princeton University Press, Princeton, New York, 2002.

[15] P. Flandrin. On the spectrum of fractional Brownian motions. IEEE Transactions on Information Theory, IT-35(1):197-199, 1989.

[16] P. Flandrin. Some aspects of nonstationary signal processing with emphasis on timefrequency and time-scale methods. In J.M. Combes, A. Grossman, and Ph. Tchamitchian, editors, Wavelets, pages 68-98. Springer-Verlag, 1989.

[17] P. Flandrin. Fractional Brownian motion and wavelets. In M. Farge, J.C.R. Hung, and J.C. Vassilicos, editors, Fractals and Fourier Transforms-New Developments and New Applications. Oxford University Press, 1991.

[18] P. Flandrin. Time-Frequency/Time-scale Analysis. Academic Press, 1st edition, 1999.

[19] R. Fox and M. S. Taqqu. Large-sample properties of parameter estimates for strongly dependent stationary Gaussian time series. Ann. Statist., 14(2):517-532, 1986. ISSN 0090-5364.

[20] L. Giraitis and D. Surgailis. Central limit theorems and other limit theorems for functionals of gaussian processes. Z. Wahrsch. verw. Gebiete, 70:191-212, 1985. 
[21] L. Giraitis and M.S. Taqqu. Whittle estimator for finite-variance non-gaussian time series with long memory. The Annals of Statistics, 27(1):178-203, 1999.

[22] A.J. Lawrance and N.T. Kottegoda. Stochastic modelling of riverflow time series. J. Roy. Statist. Soc. Ser. A,, 140(1):1-47, 1977.

[23] P. Major. Multiple Wiener-Itô integrals, volume 849 of Lecture Notes in Mathematics. Springer, Berlin, 1981. ISBN 3-540-10575-1.

[24] E. Moulines, F. Roueff, and M. S. Taqqu. On the spectral density of the wavelet coefficients of long memory time series with application to the log-regression estimation of the memory parameter. J. Time Ser. Anal., 28(2):155-187, 2007.

[25] I. Nourdin and G. Peccati. Stein's method meets Malliavin calculus: a short survey with new estimates. Technical report, HAL, 2009. URL http://hal.archives-ouvertes.fr/hal-00398006/fr/. To appear in Recent Advances in Stochastic Dynamics and Stochastic Analysis.

[26] I. Nourdin and G. Peccati. Stein's method on wiener chaos. Probability Theory and Related Fields, 154(1-2):75-118, 2009.

[27] D. Nualart. The Malliavin Calculus and Related Topics. Springer, 2006.

[28] P. M. Robinson. Log-periodogram regression of time series with long range dependence. The Annals of Statistics, 23:1048-1072, 1995.

[29] P. M. Robinson. Gaussian semiparametric estimation of long range dependence. Ann. Statist., 23:1630-1661, 1995.

[30] F. Roueff and M. S. Taqqu. Central limit theorems for arrays of decimated linear processes. Stoch. Proc. App., 119(9):3006-3041, 2009.

[31] F. Roueff and M. S. Taqqu. Asymptotic normality of wavelet estimators of the memory parameter for linear processes. J. Time Ser. Anal., 30(5):534-558, 2009. doi: 10.1111/j.1467-9892.2009.00627.x. URL http://dx.doi.org/10.1111/j.1467-9892.2009.00627.x.

[32] A. Scherrer. Analyses statistiques des communications sur puce. $\mathrm{PhD}$ thesis, École normale supérieure de Lyon, $2006 . \quad$ URL http://www .ens-lyon.fr/LIP/Pub/Rapports/PhD/PhD2006/PhD2006-09.pdf.

[33] M. S. Taqqu. A representation for self-similar processes. Stoch. Proc. Appl., 7:55-64, 1978.

[34] M. S. Taqqu. Central limit theorems and other limit theorems for functionals of gaussian processes. Z. Wahrsch. verw. Gebiete, 70:191-212, 1979.

[35] G. W. Wornell and A. V. Oppenheim. Estimation of fractal signals from noisy measurements using wavelets. IEEE Trans. Signal Process., 40(3):611 - 623, March 1992.

Laboratoire Jean Kuntzmann, Université de Grenoble, CNRS, F38041 Grenoble Cedex 9

E-mail address: marianne.clausel@imag.fr

Institut Mines-Telecom, Telecom Paristech, CNRS LTCI, 46 Rue Barrault, 75634 Paris Cedex 13, FRANCE

E-mail address: roueff@telecom-paristech.fr

Departement of Mathematics and Statistics, Boston University, Boston, MA 02215, USA

E-mail address: murad@math.bu.edu

Laboratoire Paul Painlevé, UMR 8524 du CNRS, Université Lille 1, 59655 Villeneuve D’AscQ, France. Associate member: SAMM, Université de Panthéon-Sorbonne Paris 1.

E-mail address: Ciprian.Tudor@math.univ-lille1.fr 